\title{
Journal of Hydraulic Engineering
}

Discussion of "Water Distribution System Analysis: Newton-Raphson Method

Revisited" by M. Spiliotis and G. Tsakiris August 2011, Vol. 137, No. 8, pp. 852-855. DOI: 10.1061/(ASCE)HY.1943-7900.0000364

--Manuscript Draft--

\begin{tabular}{|c|c|}
\hline Manuscript Number: & HYENG-7944R1 \\
\hline Article Type: & Discussion \\
\hline Corresponding Author: & $\begin{array}{l}\text { Dejan Brkic, Ph.D. in Petroleum Eng. } \\
\text { - } \\
\text { Beograd, SERBIA }\end{array}$ \\
\hline \multicolumn{2}{|l|}{$\begin{array}{l}\text { Corresponding Author Secondary } \\
\text { Information: }\end{array}$} \\
\hline Corresponding Author's Institution: & - \\
\hline \multicolumn{2}{|l|}{ First Author Secondary Information: } \\
\hline Order of Authors: & Dejan Brkic, Ph.D. in Petroleum Eng. \\
\hline
\end{tabular}


Dear editorial staff (your additional request about tables),

I do not have any tables in my text; (Etable 1 and Etable 2 are submitted as Electronic Annexes to the discussion; do not embed them into the text, instead provide a link to this material). This is possible for technical papers, technical notes and case studies since there is separate item description in editorial system for such purposes. This item description does not exist for discussions. So I have uploaded them as figures. Please, advise me if you have additional requests with these materials.

Dear Editor/Reviewers,

I have accepted all your suggestions. I am not native English speaker and therefore I appreciate your grammar corrections of my text. Thank you!

My text is:

Discussion of "Water Distribution System Analysis: Newton-Raphson Method Revisited" by M. Spiliotis and G. Tsakiris

August 2011, Vol. 137, No. 8, pp. 852-855.

DOI: 10.1061/(ASCE)HY.1943-7900.0000364

My discussion contains Supplemental Data; Electronic tables, E-table 1 and E-table 2 (the Excel files with calculations converted in PDF). They can be attached to the electronic version of the discussion in the ASCE Library.

Sincerely yours,

Dejan Brkić, Ph.D. 
1 Discussion of "Water Distribution System Analysis: Newton-Raphson Method

2 Revisited" by M. Spiliotis and G. Tsakiris

3 August 2011, Vol. 137, No. 8, pp. 852-855.

4 DOI: 10.1061/(ASCE)HY.1943-7900.0000364

5 Dejan Brkić, Ph.D., Petroleum and Natural Gas Engineer, Strumička 88, 11050 Belgrade,

6 Serbia, e-mail: dejanrgf@tesla.rcub.bg.ac.rs, dejanbrkic0611@gmail.com

Spiliotis and Tsakiris (2011) show the nodal formulation for water distribution system analysis using the head-loss dependent Jacobian. Some issues about the node formulation of

the discussed problem will be commented on here. Also, as an addition to the discussed paper, the procedure with pressure drop formulation of the Jacobian will be presented where the method is similar to, but different from Spiliotis and Tsakiris (2011). The procedure uses elements of the methods by Cross (1936), Shamir and Howard (1968) and Epp and Fowler (1970).

As shown in Brkić (2011a), all methods for the analysis of looped water distribution systems can be divided in two general groups: loop oriented and node oriented methods. To date, all loop oriented methods contained a flow independent Jacobian while all node oriented methods contained a pressure drop (or a head loss) independent Jacobian. In their recent paper, Simpson and Elhay (2011) show an improved loop method with the flow-dependent Jacobian where the problem is how to find the derivative of the implicitly given equation by Colebrook (1939) which is in a common use as a standard for the calculation of flow friction, $\lambda$ [the Colebrook equation is Eq. $1 \mathrm{~b}$ in the discussed paper (the Darcy friction is noted as $\mathrm{f}$ in the discussed paper and in this discussion as $\lambda$ )]. The problem can be addressed by using some of the available explicit approximations of the Colebrook equation [Swamee and Jain (1976) 
approximation is used in Simpson and Elhay (2011)], but as shown in Brkić (201x) it is not always possible to find a balance between accuracy and complexity among many available approximate formulas (Brkić 2011b). On the other hand, Spiliotis and Tsakiris (2011) show the node oriented method with the head-loss dependent Jacobian. In general, the convergence properties of the nodal formulation are not as good as those of the looped version, but, on the other hand, the derivative of the implicitly given Colebrook equation used in the proposed pressure drop or head-loss dependent Jacobian can be easily calculated when the nodal method is employed. To accomplish the solution of the problem in the pressure drop dependent form, Eq. 13, is used for non-iterative calculation of flow Q.

$$
F=Q=\pi \cdot \sqrt{\frac{\Delta p \cdot D^{5}}{2 \cdot L \cdot \rho}} \cdot \log \left(\frac{2.51 \cdot v \cdot \sqrt{L \cdot \rho}}{\sqrt{2 \cdot \Delta p \cdot D^{3}}}+\frac{\varepsilon}{3.7 \cdot D}\right)
$$

Eq. 13 is derived combining the Darcy-Weisbach equation, $\lambda=\Delta p \cdot \pi^{2} \cdot D^{5} /\left(8 \cdot L \cdot \rho \cdot Q^{2}\right)$, and the equation for the Reynolds number, $\mathrm{Re}=4 \cdot \mathrm{Q} /(\mathrm{D} \cdot \pi \cdot v)$ as in Eq. 14 , and then combining the Colebrook equation and again the Darcy-Weisbach equation to produce Eq. 13.

$\operatorname{Re} \cdot \sqrt{\lambda}=\frac{4 \cdot Q}{D \cdot \pi \cdot v} \cdot \sqrt{\frac{\Delta p \cdot \pi^{2} \cdot D^{5}}{8 \cdot L \cdot \rho \cdot Q^{2}}}=\frac{1}{v} \cdot \sqrt{\frac{2 \cdot \Delta p \cdot D^{3}}{L \cdot \rho}}$

In that way, friction factor, $\lambda$, is eliminated from Eq. 13 which implies that flow, Q, in a single pipe can be calculated using the implicit Colebrook equation but also at the same time, avoiding iteration (Swamee and Jain 1976). On the contrary, standard procedure requires the iterative calculation of flow friction $\lambda$, using the implicitly given Colebrook equation [Eq. $1 \mathrm{~b}$ of the discussed paper] and then the Darcy-Weisbach equation rearranged for calculation of flow $\mathrm{Q}=\left[\Delta \mathrm{p} \cdot \pi^{2} \cdot \mathrm{D}^{5} /(8 \cdot \mathrm{L} \cdot \rho \cdot \lambda)\right]^{0.5}$. However, a calculation of flow (or head, i.e. pressure distribution) in a looped system of pipes still requires an iterative procedure, but by using Eq. 13, a double iterative procedure can be avoided (an iterative procedure for the calculation of a looped system of pipes still exists while the sub-iterative calculation of a single pipe in a 
looped system required by the Colebrook equation is not needed). Of course, Eq. 13 has additional value because it can be used for the calculation of the partial derivative $\mathrm{F}^{\prime}=\partial \mathrm{Q}(\Delta \mathrm{p}) / \partial(\Delta \mathrm{p})$, where pressure drop $\Delta \mathrm{p}$ is treated as the variable.

Appropriate partial derivatives, F', are the terms in the pressure drop dependent Jacobian matrix [matrix $[\mathrm{J}]$ in Eq. 15 of this discussion (the relevant head-loss dependent Jacobian matrix is presented by Eq. 9 in the discussed paper)].

As noted in the discussed paper, this calculation is simple and hence can be performed in Excel. An Excel file converted to PDF are accompanied as an electronic annex to this discussion (Electronic tables; E-tables 1 and 2) [the example network (shown in Fig. 1 of the discussed paper is used as the example]. In this discussion, two versions of the standard procedure with a standard pressure drop independent Jacobian are shown (in E-tables 1 and 2) where convergence properties of both are investigated. The presented procedures start from a pressure-drop distribution which satisfies the second Kirchhoff law for all closed paths in the network of pipes and which is iterated until continuity at each node is approximately satisfied. Initial choices for pressure drops are; for the first loop: $\Delta \mathrm{p}_{12}=1000 \mathrm{kPa}, \Delta \mathrm{p}_{25}=500 \mathrm{kPa}, \Delta \mathrm{p}_{56}=-$ $750 \mathrm{kPa}, \Delta \mathrm{p}_{61}=-750 \mathrm{kPa}$; and for the second loop: $\Delta \mathrm{p}_{52}=-500 \mathrm{kPa}, \Delta \mathrm{p}_{23}=600 \mathrm{kPa}, \Delta \mathrm{p}_{34}=-600 \mathrm{kPa}$ and $\Delta \mathrm{p}_{45}=500 \mathrm{kPa}$ [direction of both loops is counterclockwise, i.e. positive]. Also, as shown in Brkić (2011a), the standard procedure with pressure drop independent Jacobian demands assumption of the velocity of water (here $10 \mathrm{~m} / \mathrm{s}$ ) required for the Reynolds number, Re, at the start of the procedure where the velocity can be chosen at random in a reasonable range without causing any trouble [the Reynolds number, Re, can be treated as independent of flow (as in E-table 1) or calculated in each iteration using flow $\mathrm{Q}$ from the previous iteration (as in E-table 2)]. On the contrary, assumption of the starting velocity is sufficient for the procedure shown in this discussion [using Eq. 13 and related derivatives F'] and in the discussed paper 
(but note that the Reynolds number, velocity and viscosity still remain connected). The choice of initial pressure drop is not critical but should satisfy the second Kirchhoff law (Gay and Middleton 1971). Kinematic viscosity of water, $v$, is $1.00410^{-6} \mathrm{~m}^{2} / \mathrm{s}$, water density, $\rho$ is $1000 \mathrm{~kg} / \mathrm{m}^{3}$ and pipe roughness coefficient, $\varepsilon$ is $310^{-6} \mathrm{~m}$ [inner roughness of the third generation polyethylene (PE) pipes]. Pipe lengths $\left(\mathrm{L}_{12}=\mathrm{L}_{45}=\mathrm{L}_{56}=400 \mathrm{~m}, \mathrm{~L}_{16}=\mathrm{L}_{25}=900 \mathrm{~m}\right.$, $\mathrm{L}_{23}=500 \mathrm{~m}$, and $\left.\mathrm{L}_{34}=800 \mathrm{~m}\right)$, pipe diameters $\left(\mathrm{D}_{12}=0.3526 \mathrm{~m}, \mathrm{D}_{16}=\mathrm{D}_{34}=0.2204 \mathrm{~m}\right.$, $\mathrm{D}_{23}=\mathrm{D}_{25}=0.2468 \mathrm{~m}, \mathrm{D}_{45}=0.1102 \mathrm{~m}$, and $\left.\mathrm{D}_{56}=0.0968 \mathrm{~m}\right)$ and water demands $\left(\mathrm{q}_{1}=-130 \mathrm{~L} / \mathrm{s}\right.$ [input in network], $\mathrm{q}_{2}=15 \mathrm{~L} / \mathrm{s}, \mathrm{q}_{3}=10 \mathrm{~L} / \mathrm{s}, \mathrm{q}_{4}=35 \mathrm{~L} / \mathrm{s}, \mathrm{q}_{5}=40 \mathrm{~L} / \mathrm{s}$, and $\mathrm{q}_{6}=30 \mathrm{~L} / \mathrm{s}$ ) are from Fig. 1 of the discussed paper and in the electronic annex to this discussion [numbers in the index are related to nodes (first number in the index is the starting node and the second is the ending node)]. The network is supplied by water through node 1 where the pressure is $1962 \mathrm{kPa}$ [node 5 is chosen to be excluded as redundant from the Jacobian matrix to preserve linear independency of rows and columns in the matrix (node 5 is chosen at random, i.e. any other node can be chosen instead of node 5; node 1 is chosen in the discussed paper)]. The calculated corrections of pressure drops in iteration i can be calculated as $\Delta x=\Delta(\Delta p)$, Eq. 15 $\left([\mathrm{J}] \mathrm{x}[\Delta \mathrm{x}]=[\mathrm{F}]\right.$, where: $[\mathrm{J}]=\left[\mathrm{F}^{\prime}\right]$-the Jacobian matrix (here with symmetrical properties, diagonal terms are positive and non-diagonal terms are negative); $[\Delta \mathrm{x}]$-single column matrix of corrections and [F]-single column matrix of flows where its terms are calculated using Eq. 13). The corrections have to be algebraically added to the values of pressure drop in the appropriate pipes in iteration i-1 [in the zeroth iteration the initial pressure drop pattern (initial choices) are chosen as explained above]. Algebraic rules for the corrections can be seen in Brkić (2009, 2011a) and in Corfield et al. (1974). 


\begin{tabular}{|c|c|c|c|c|c|c|}
\hline & & & & & {$[\langle x]$} & {$[\underbrace{F]}$} \\
\hline$F_{1}^{\prime}\left(\left|-x_{12}\right|,\left|-x_{16}\right|\right)$ & $-\left|F_{1}^{\prime}\left(x_{12}\right)\right|$ & 0 & 0 & $-\left|F_{1}^{\prime}\left(x_{16}\right)\right|$ & $\Delta x_{1}$ & $-\mathrm{Q}_{12}-\mathrm{Q}_{16}+q_{1}$ \\
\hline$-\left|F_{2}^{\prime}\left(x_{21}\right)\right|$ & $F_{2}^{\prime}\left(\left|+x_{21}\right|,\left|-x_{23}\right|,\left|-x_{25}\right|\right)$ & $-\left|F_{2}^{\prime}\left(x_{23}\right)\right|$ & 0 & 0 & $\Delta x_{2}$ & $+\mathrm{Q}_{21}-\mathrm{Q}_{23}-\mathrm{Q}_{25}-q_{2}$ \\
\hline 0 & $-\left|F_{3}^{\prime}\left(x_{32}\right)\right|$ & $F_{3}^{\prime}\left(\left|+x_{32}\right|,\left|+x_{34}\right|\right)$ & $-\left|F_{3}^{\prime}\left(x_{34}\right)\right|$ & 0 & \begin{tabular}{l|l}
$x$ & $\Delta x_{3}$
\end{tabular} & $=\quad+\mathrm{Q}_{32}+\mathrm{Q}_{34}-q_{3}$ \\
\hline 0 & 0 & $-\left|F_{4}^{\prime}\left(x_{43}\right)\right|$ & $F_{4}^{\prime}\left(\left|-x_{43}\right|,\left|-x_{45}\right|\right)$ & 0 & $\Delta x_{4}$ & $-\mathrm{Q}_{43}-\mathrm{Q}_{45}-q_{4}$ \\
\hline$-\left|F_{6}^{\prime}\left(x_{61}\right)\right|$ & 0 & 0 & 0 & $F_{6}^{\prime}\left(\left|+x_{61}\right|,\left|-x_{65}\right|\right)$ & $\left\lfloor x_{6}\right\rfloor$ & $+\mathrm{Q}_{61}-\mathrm{Q}_{65}-q_{6}$ \\
\hline
\end{tabular}
(15)

Standard nodal oriented calculation (with flow independent Jacobian) can be with a constant value of the Reynolds number, $\operatorname{Re}[$ velocity, $\mathrm{v}=$ const $\rightarrow \mathrm{Re}=\mathrm{v} \cdot \mathrm{D} / \mathrm{v}$ ] and with the flow dependent version $\left[\mathrm{v}=4 \cdot \mathrm{Q} /\left(\mathrm{D}^{2} \cdot \pi\right) \rightarrow \mathrm{Re}=\mathrm{f}(\mathrm{Q})\right]$ (shown in E-tables 1 and 2 , respectively). Final calculated pressure drops and related flows after 7 iterations for a constant value of the Reynolds number (E-table 1) are $\Delta \mathrm{p}_{12}=-5559 \mathrm{kPa}, \mathrm{Q}_{12}=-97.1 \mathrm{~L} / \mathrm{s} ; \Delta \mathrm{p}_{16}=-16244 \mathrm{kPa}, \mathrm{Q}_{16}=-$ $32.9 \mathrm{~L} / \mathrm{s} ; \Delta \mathrm{p}_{23}=-7566 \mathrm{kPa}, \mathrm{Q}_{23}=-40.3 \mathrm{~L} / \mathrm{s} ; \Delta \mathrm{p}_{25}=-14612 \mathrm{kPa}, \mathrm{Q}_{25}=-41.8 \mathrm{~L} / \mathrm{s} ; \Delta \mathrm{p}_{34}=-12287 \mathrm{kPa}$, $\mathrm{Q}_{34}=-30.3 \mathrm{~L} / \mathrm{s} ; \Delta \mathrm{p}_{45}=5241 \mathrm{kPa}, \mathrm{Q}_{45}=4.7 \mathrm{~L} / \mathrm{s} ;$ and $\Delta \mathrm{p}_{56}=3926 \mathrm{kPa}, \mathrm{Q}_{56}=2.9 \mathrm{~L} / \mathrm{s}$ [where velocity used for the calculation of the Reynolds number does not change between two iterations (velocity in E-table 1 is $10 \mathrm{~m} / \mathrm{s}$ in all iterations, tests with a value of randomly chosen velocity between $0.1 \mathrm{~m} / \mathrm{s}$ and $500 \mathrm{~m} / \mathrm{s}$ resulted in only a minor influence on the final results)]. On the other hand, when calculation with the flow dependent Reynolds number takes place, results are similar but slightly different. Results after 12 iterations are (E-table 2): $\Delta \mathrm{p}_{12}=-7983 \mathrm{kPa}$, $\mathrm{Q}_{12}=-97.4 \mathrm{~L} / \mathrm{s} ; \Delta \mathrm{p}_{16}=-23769 \mathrm{kPa}, \mathrm{Q}_{16}=-32.6 \mathrm{~L} / \mathrm{s} ; \Delta \mathrm{p}_{23}=-11343 \mathrm{kPa}, \mathrm{Q}_{23}=-40.5 \mathrm{~L} / \mathrm{s} ; \Delta \mathrm{p}_{25}=-$ $21696 \mathrm{kPa}, \mathrm{Q}_{25}=-41.9 \mathrm{~L} / \mathrm{s} ; \Delta \mathrm{p}_{34}=-18740 \mathrm{kPa}, \mathrm{Q}_{34}=-30.5 \mathrm{~L} / \mathrm{s} ; \Delta \mathrm{p}_{45}=8387 \mathrm{kPa}, \mathrm{Q}_{45}=4.5 \mathrm{~L} / \mathrm{s} ;$ and $\Delta \mathrm{p}_{56}=5910 \mathrm{kPa}, \mathrm{Q}_{56}=2.6 \mathrm{~L} / \mathrm{s}$. The last results are maybe more accurate, but using the same stopping criterion for comparison, the calculation with the flow-dependent Reynolds number does not fulfilled the required accuracy even after 12 iterations (in E-table 2, calculation of the Reynolds number also starts with a velocity of $10 \mathrm{~m} / \mathrm{s}$ but then the velocity for the next iteration is calculated using value of flow, Q, from the previous iteration). To conclude, as can be seen from Fig. 13, a less sophisticated method with a constant value of the Reynolds number requires less iterative cycles (but maybe produces less reliable results). Every new method should be employed with caution and tested with a wide range of criteria (not only 
with the required number of iterations). Therefore, the new method with pressure drop and head-loss dependent Jacobian should be further evaluated (to avoid unfounded conclusions).

Figure 1. Convergence properties of the proposed method for the solution of the problem of water distribution analysis expressed in the nodal form (both with the independent Jacobian)

As can be seen from Eqs. 15 [and Eqs. 5 and 10 of the discussed paper], the procedure with the pressure drop dependent Jacobian [or head-loss dependent one from the discussed paper], does not require calculation of the Reynolds number at all (problem from E-tables 1 and 2 is exceeded). On the other hand, Eqs. 15 [and Eq. 4 and 5 of the discussed paper] and its derivative $F^{\prime}$ [Eq. 10 of the discussed paper] are complex for computing by hand but they can be easily implemented in a computer code.

In addition to the discrepancy in results from the use of a dependent versus independent Jacobian matrix, a portion of the discrepancy in results of this discussion (in addition to the fact that the discussed paper head, $\mathrm{h}$ is in $\mathrm{m}$, and in this discussion pressure drops $\Delta \mathrm{p}$ are in $\mathrm{Pa}$, where $\Delta \mathrm{p}=\rho \cdot \mathrm{g} \cdot \mathrm{h}$ ) compared with the discussed paper is probably caused by use of different values for the roughness of pipes, $\varepsilon$, and kinematic viscosity of water, $v$ [Spiliotis and Tsakiris (2011) did not report these values].

\section{Supplemental Data}

The Excel files converted in PDF (Electronic tables; E-tables 1 and 2) used as examples in this discussion is available online in the ASCE Library (www.ascelibrary.org)

\section{Notation}


145 The following symbols are used in this discussion:

146 D inner pipe diameter $(m)$

147 L pipe length (m)

148 Q flow rate $\left(\mathrm{m}^{3} / \mathrm{s}\right)$

$149 \mathrm{q} \quad$ water demand $\left(\mathrm{m}^{3} / \mathrm{s}\right)$

150 Re Reynolds number (-)

$151 \Delta \mathrm{p} \quad$ pressure drop $(\mathrm{Pa})$

$152 \Delta \mathrm{x} \quad$ correction of pressure drop $(\mathrm{Pa})$

$153 \varepsilon \quad$ pipe roughness coefficient (m)

$154 \lambda \quad$ Darcy (Darcy-Weisbach) or Moody friction factor (-)

$155 \quad v \quad$ kinematic viscosity $\left(\mathrm{m}^{2} / \mathrm{s}\right)$

$156 \rho \quad$ water density $\left(\mathrm{kg} / \mathrm{m}^{3}\right)$

157

158 References:

159 Brkić, D. (2009), “An improvement of Hardy Cross method applied on looped spatial natural 160 gas distribution networks.” Appl.Energ. 86(7-8), 1290-1300.

161 Brkić, D. (2011a), "Iterative methods for looped network pipeline calculation," Water Resour 162 Manag. 25(12), 2951-2987.

163 Brkić, D. (2011b), "Review of explicit approximations to the Colebrook relation for flow 164 friction," J. Petrol. Sci.Eng., 77(1), 34-48.

165 Brkić, D. (201x), "Discussion of 'Jacobian matrix for solving water distribution system 166 equations with the Darcy-Weisbach head-loss model'," J. Hydraul. Eng. ASCE, (in press) doi: 167 10.1061/(ASCE)HY.1943-7900.0000529 
Corfield, G., Hunt, B.E., Ott, R.J., Binder, G.P., Vandaveer, F.E. (1974), “Distribution design for increased demand". In: Segeler, C.G. (Editor): Gas Engineers Handbook. Industrial Press, New York, Chapter 9, 63-83.

Colebrook, C. F. (1939). "Turbulent flow in pipes with particular reference to the transition region between the smooth and rough pipe laws," J. Inst. Civil. Eng.(London), 11(4), 133156.

Cross, H. (1936). "Analysis of flow in networks of conduits or conductors," University of Illinois at Urbana Champaign, College of Engineering. Engineering Experiment Station 286(34), 3-29. http://hdl.handle.net/2142/4433

Epp, R, and Fowler, A.G. (1970). "Efficient code for steady flows in networks," J. Hydraul. Div. ASCE, 96(1), 43-56.

Gay, B, and Middleton, P. (1971). "The solution of pipe network problems," Chem. Eng. Sci., 26(1), 109-123.

Shamir, U., and Howard, C. (1968). "Water distribution system analysis," J. Hydraul. Div. ASCE, 94(1), 219-234.

Simpson, A., and Elhay, S. (2011). “Jacobian matrix for solving water distribution system equations with the Darcy-Weisbach head-loss model," J. Hydraul. Eng. ASCE, 137 (6), 696700.

Spiliotis, M. and Tsakiris, G. (2011). "Water distribution system analysis: Newton-Raphson method revisited," J. Hydraul. Eng. ASCE, 137 (8), 852-855.

Swamee, P. K., and Jain, A. K. (1976). "Explicit equations for pipe-flow problems,” J. Hydraul.Div. ASCE, 102(5), 657-664. 
Figure $1 \mathrm{FB}$

Click here to download high resolution image

$\mathrm{Q}\left[\mathrm{dm}^{3} / \mathrm{s}\right]$

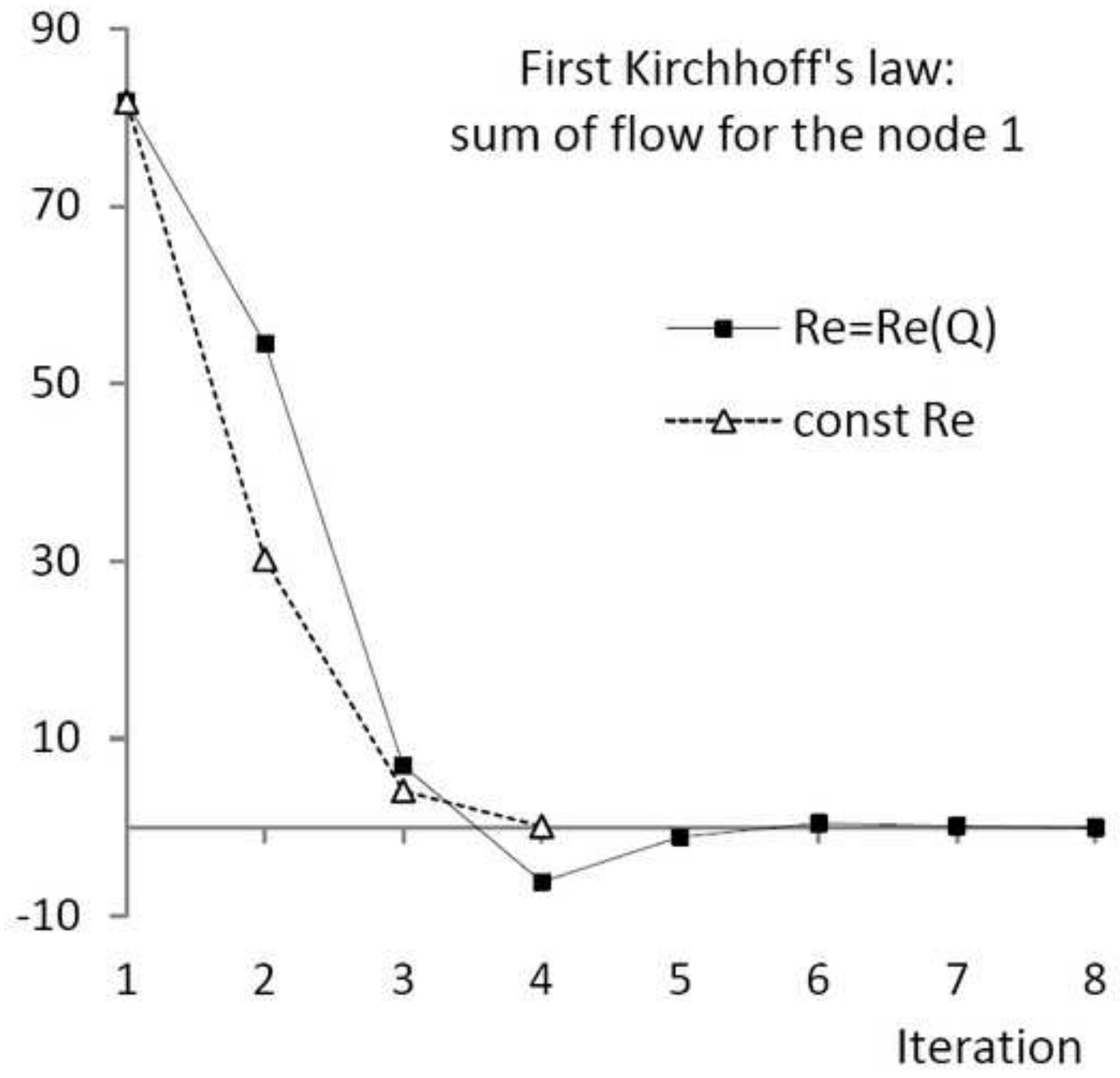




\section{Parameters of network}

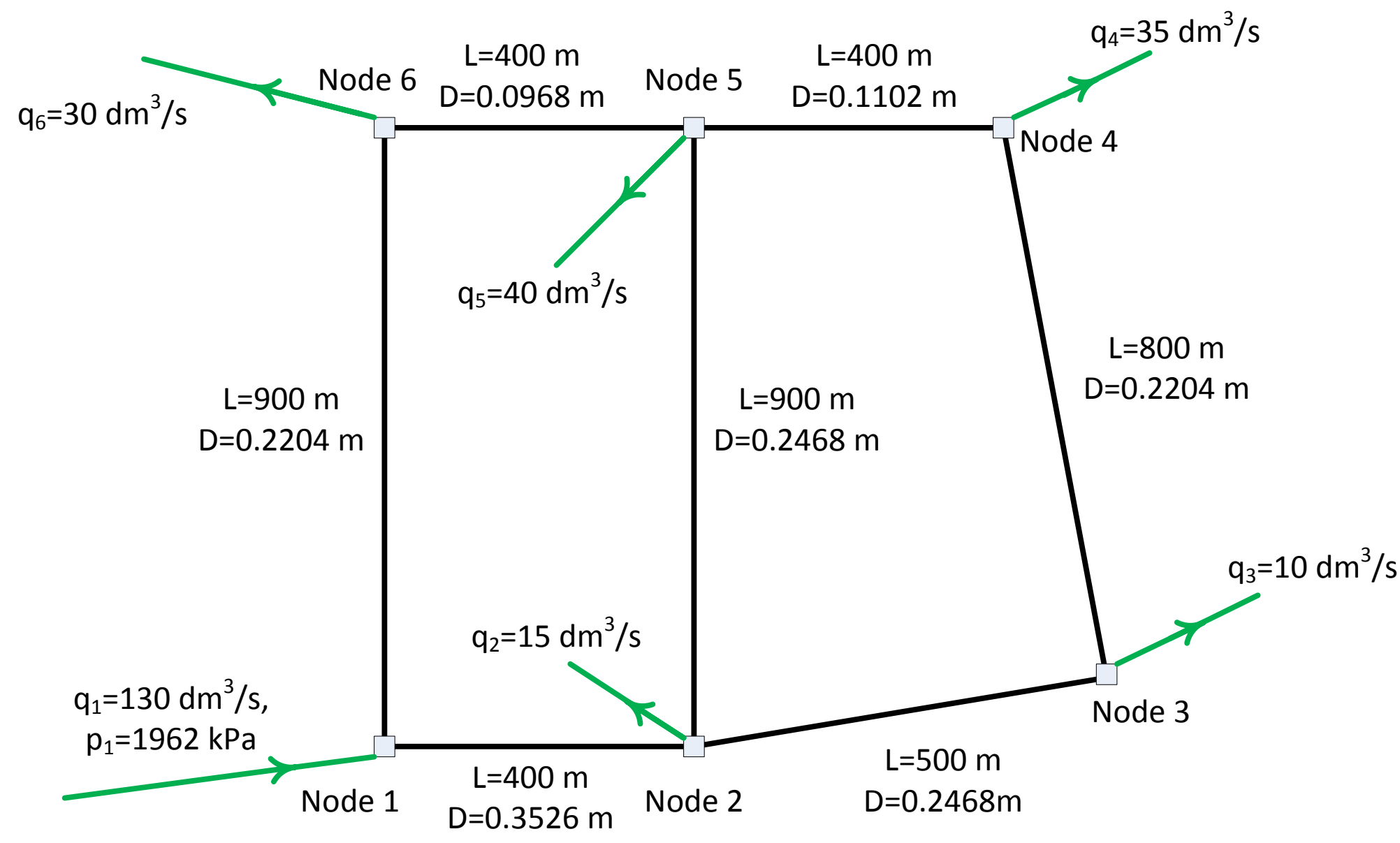

\section{L-length}

D-diameter

q-water demands and input, Q-flows

p-pressure, $\Delta p$-pressure drop

$\varepsilon$-roughness of pipes; $\varepsilon=3 \cdot 10^{-6} \mathrm{~m}$

$v$-kinematic viscosity of water; $v=1.004 \cdot 10^{-6} \mathrm{~m}^{2} / \mathrm{s}$

$\rho$-water density; $\rho=1000 \mathrm{~kg} / \mathrm{m}^{3}$

Final pattern of pressure (after 7 iterations)

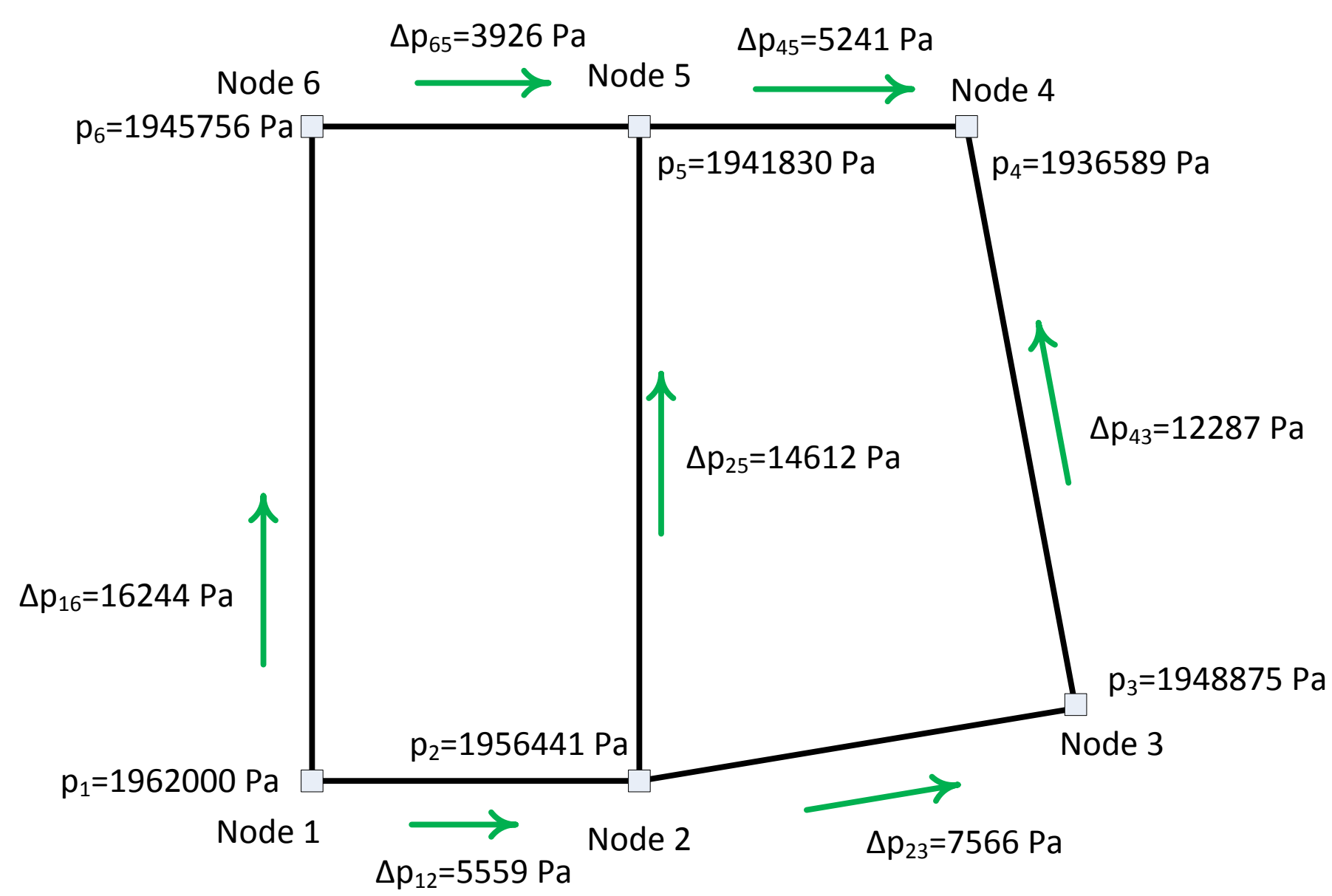

Initial pattern of pressure drops

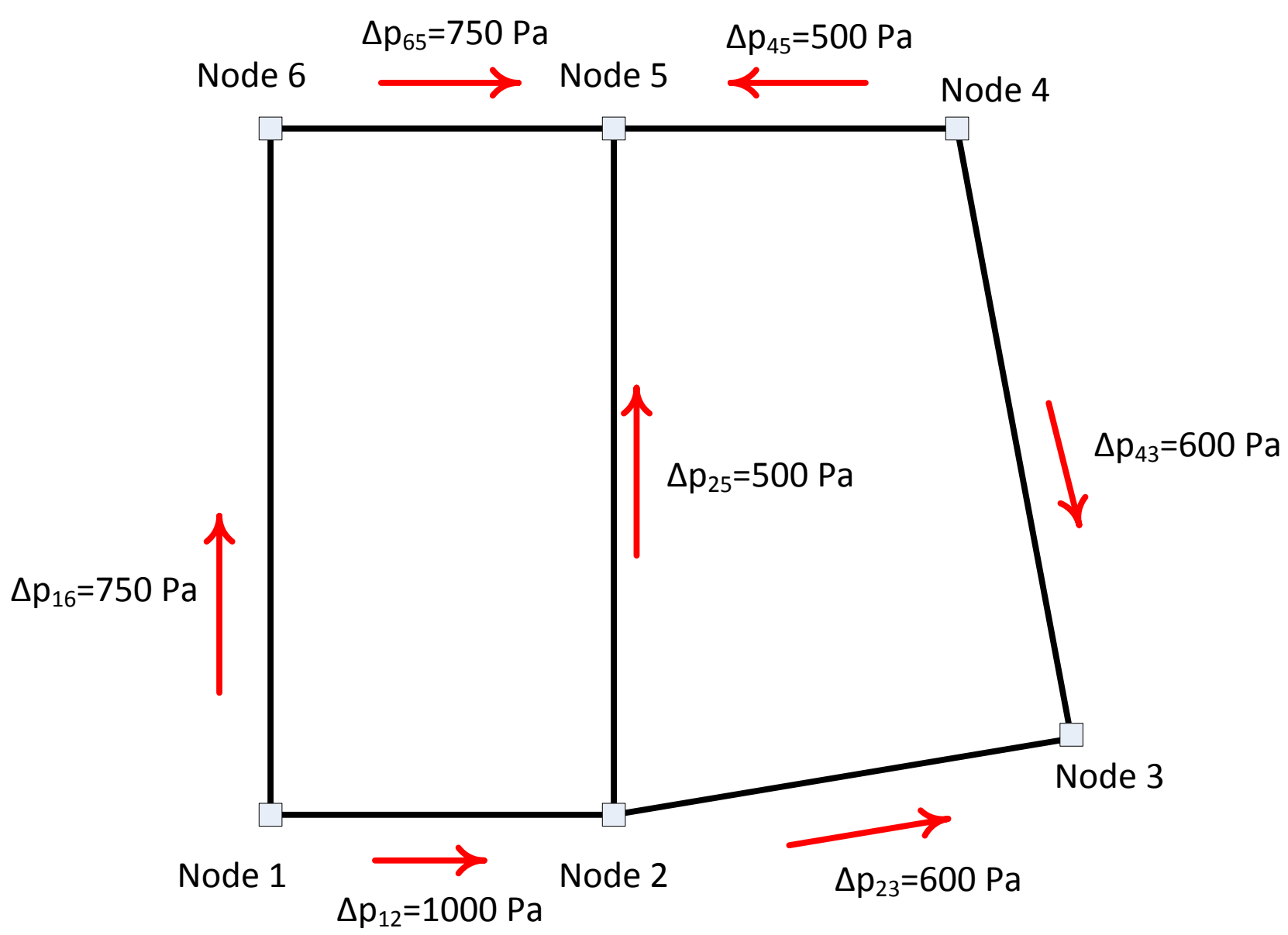

Final flows (after 7 iterations)

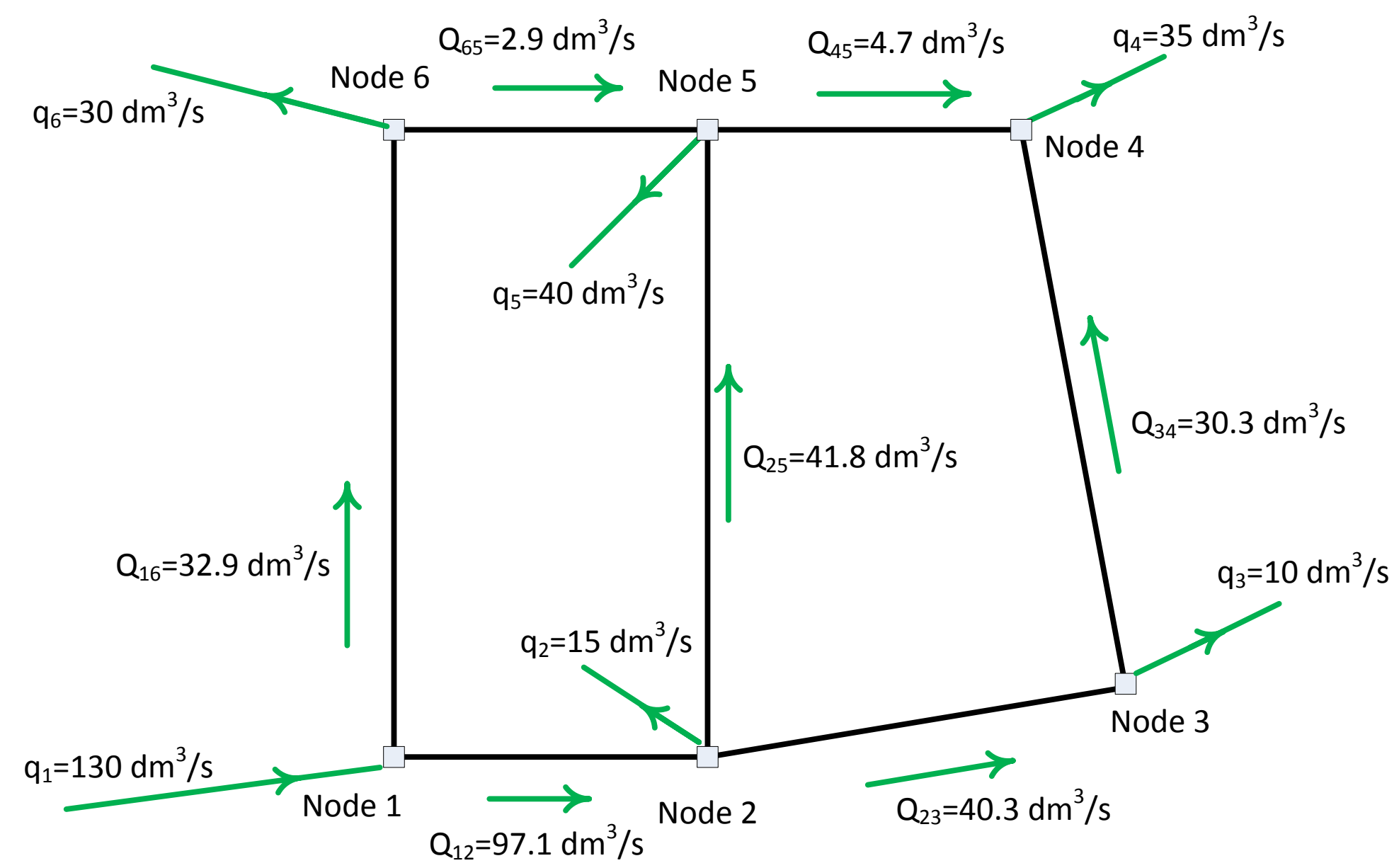


Standard procedure (pressure drop independent Jacobian); Re-const

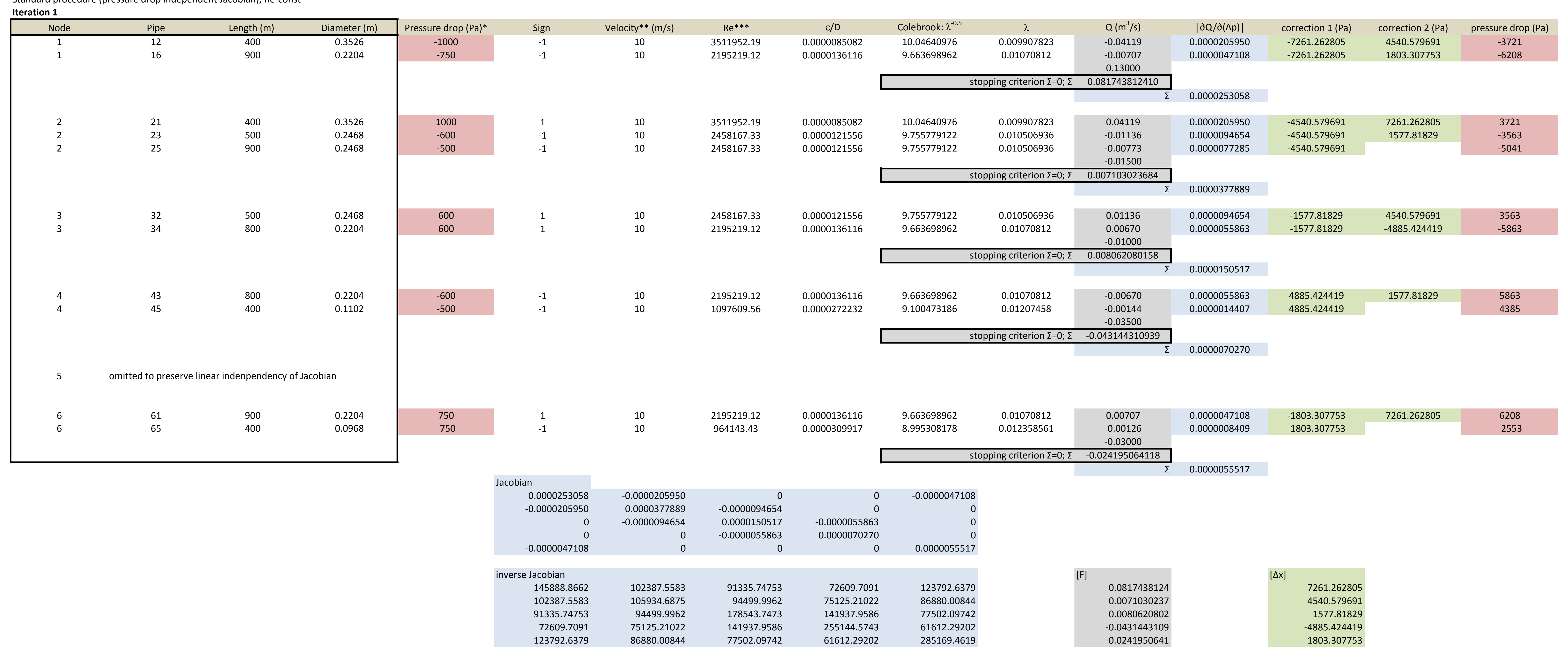


Standard procedure (pressure drop independent Jacobian); Re-const

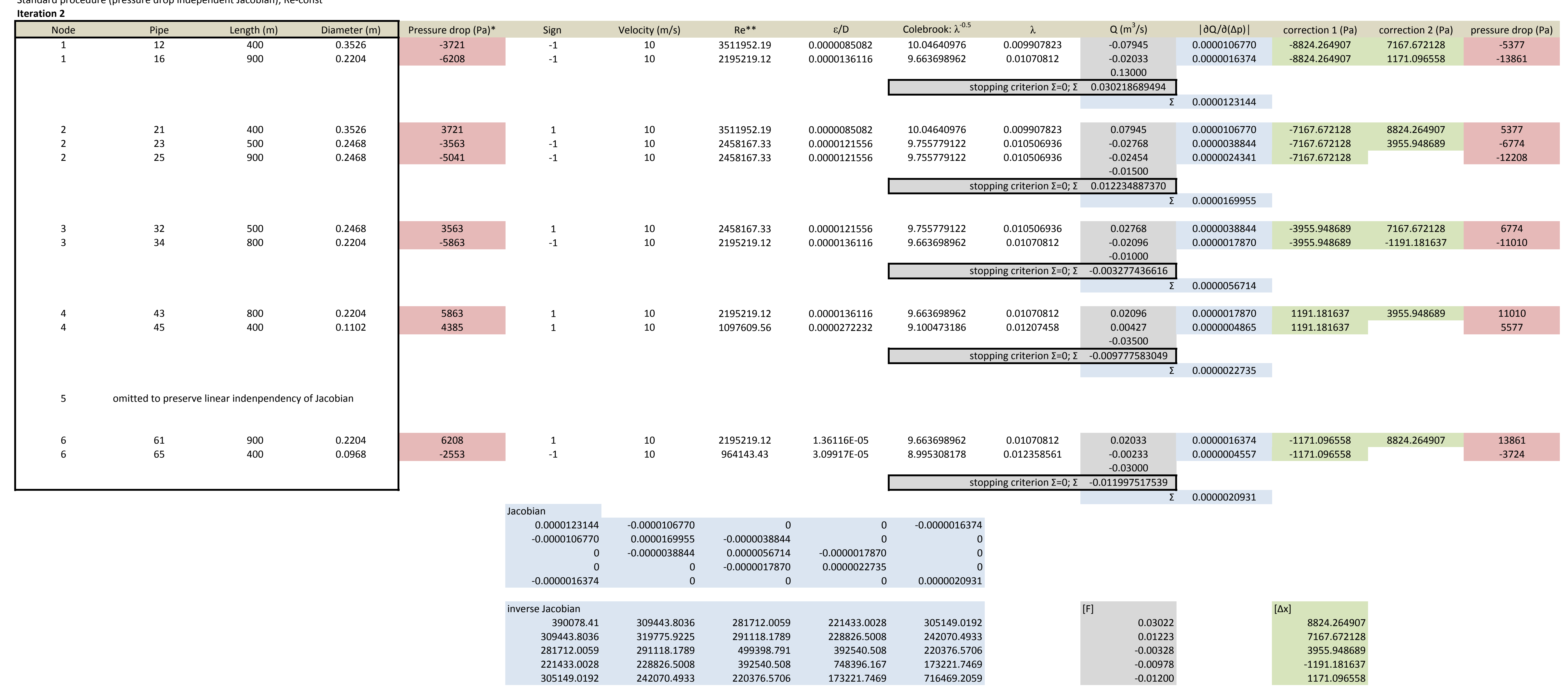

"from the previous iteration
$*$ *constant value of the Reynolds number

Dejan Brkic, Strumicka 88, 11050 Belgrade, Serbia; dejanrg@@tesla.rcub.bg.ac.rs, dejanbrkic0611@gmail.com 
Standard procedure (pressure drop independent Jacobian); Re-const

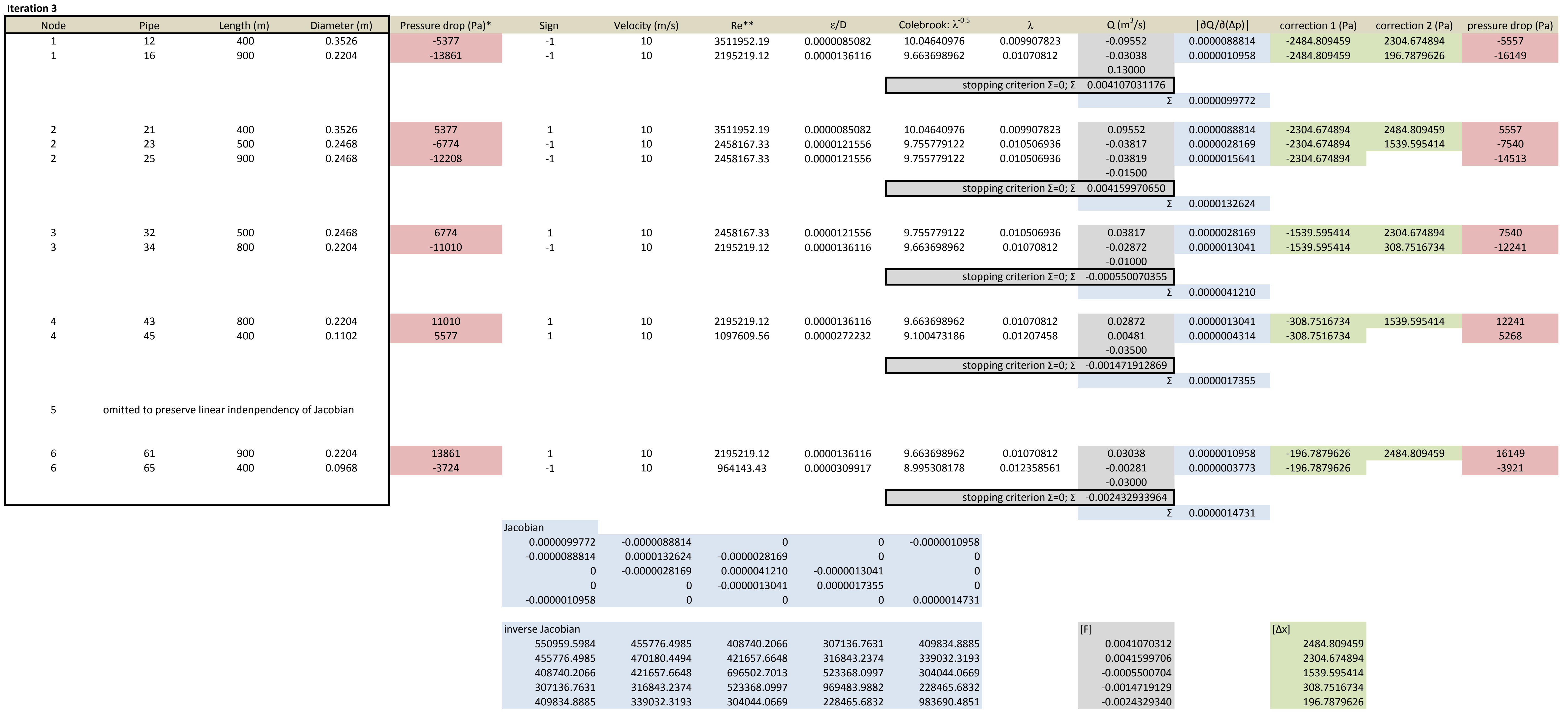




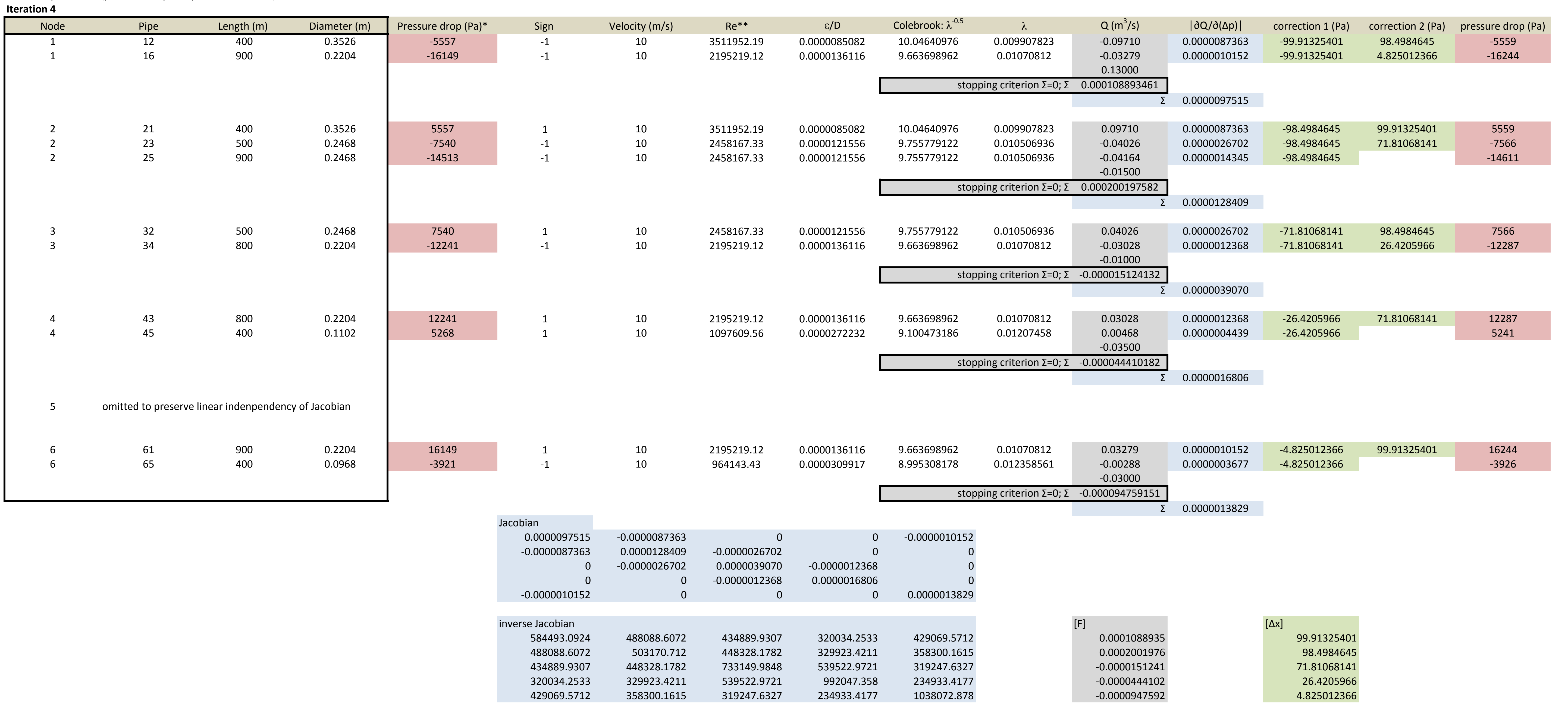




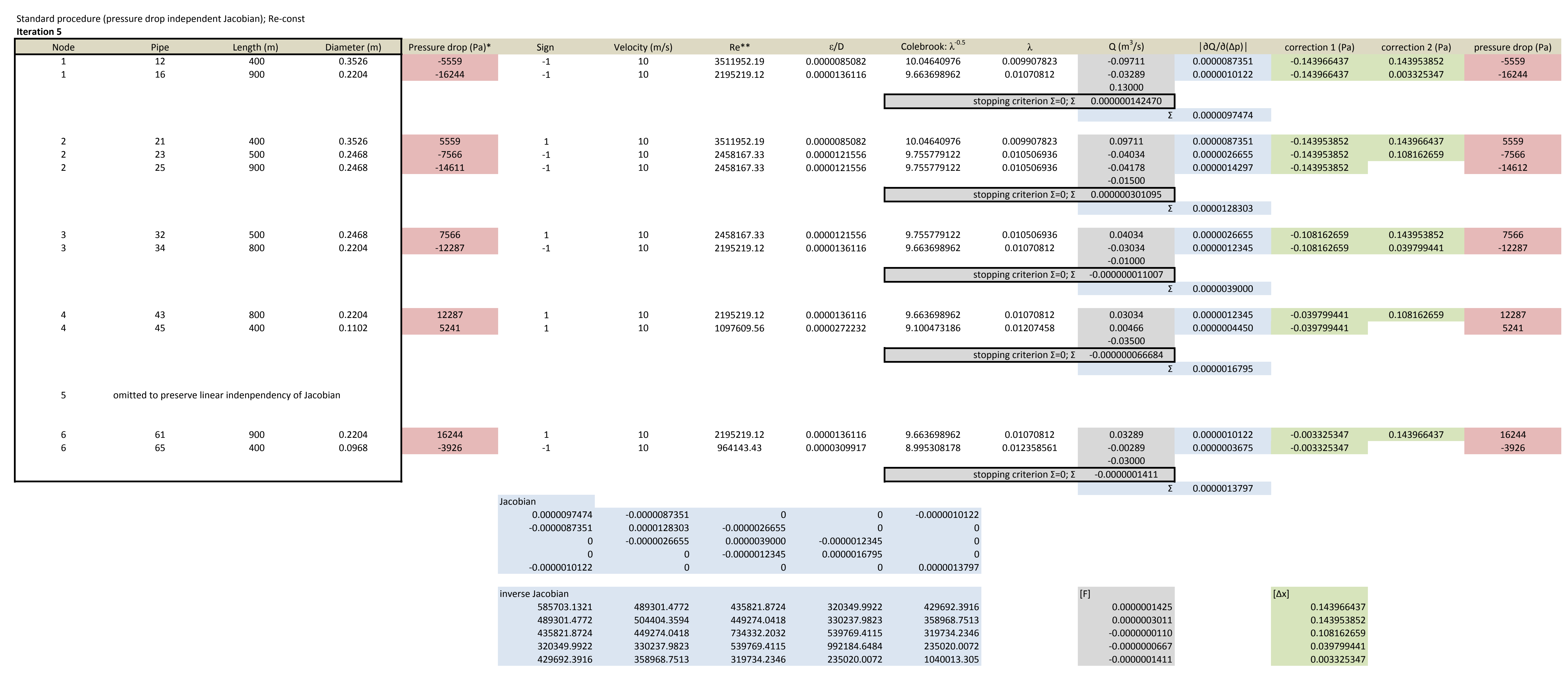

${ }^{*}$ from the previous iteration
** constant value of the Reynolds number $^{2}$

Dejän Brktic, Strumicka R8, 11050 Belgrade, Serbia; dejanref@@esla.rcub.bg.ac.rs, dejanbrkic0611@gmail.com 
Standard procedure (pressure drop independent Jacobian); Re-const

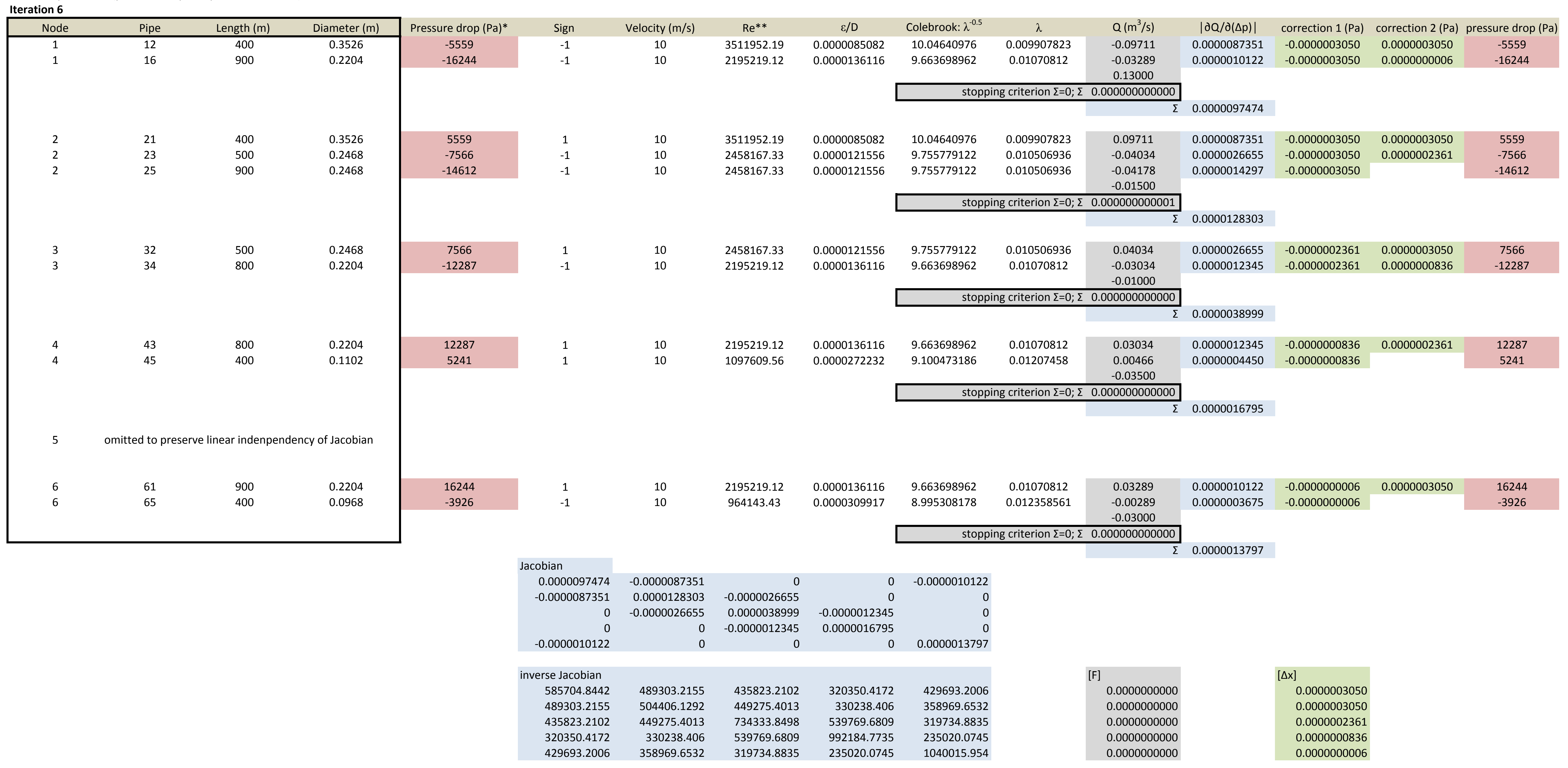

*from the previous iteration

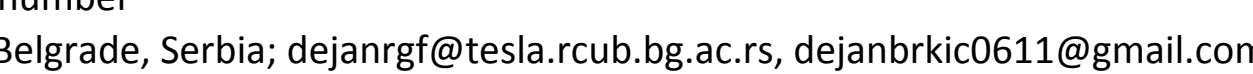




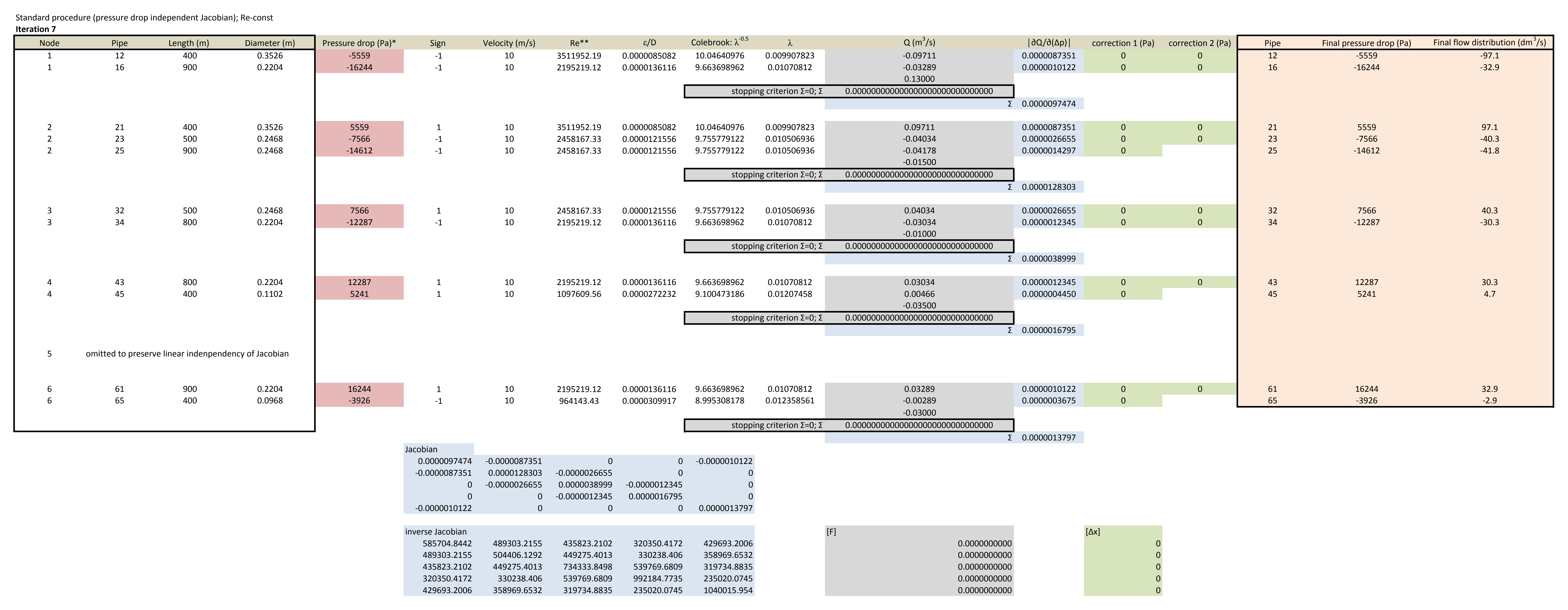

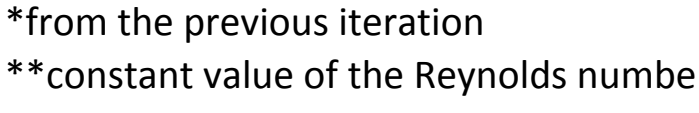

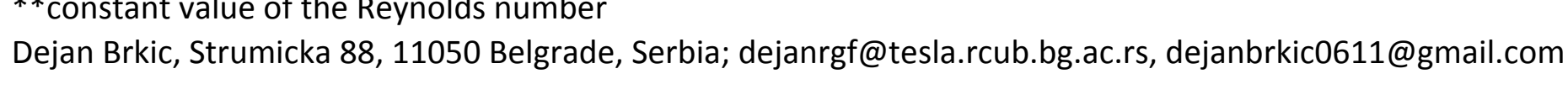


Parameters of network

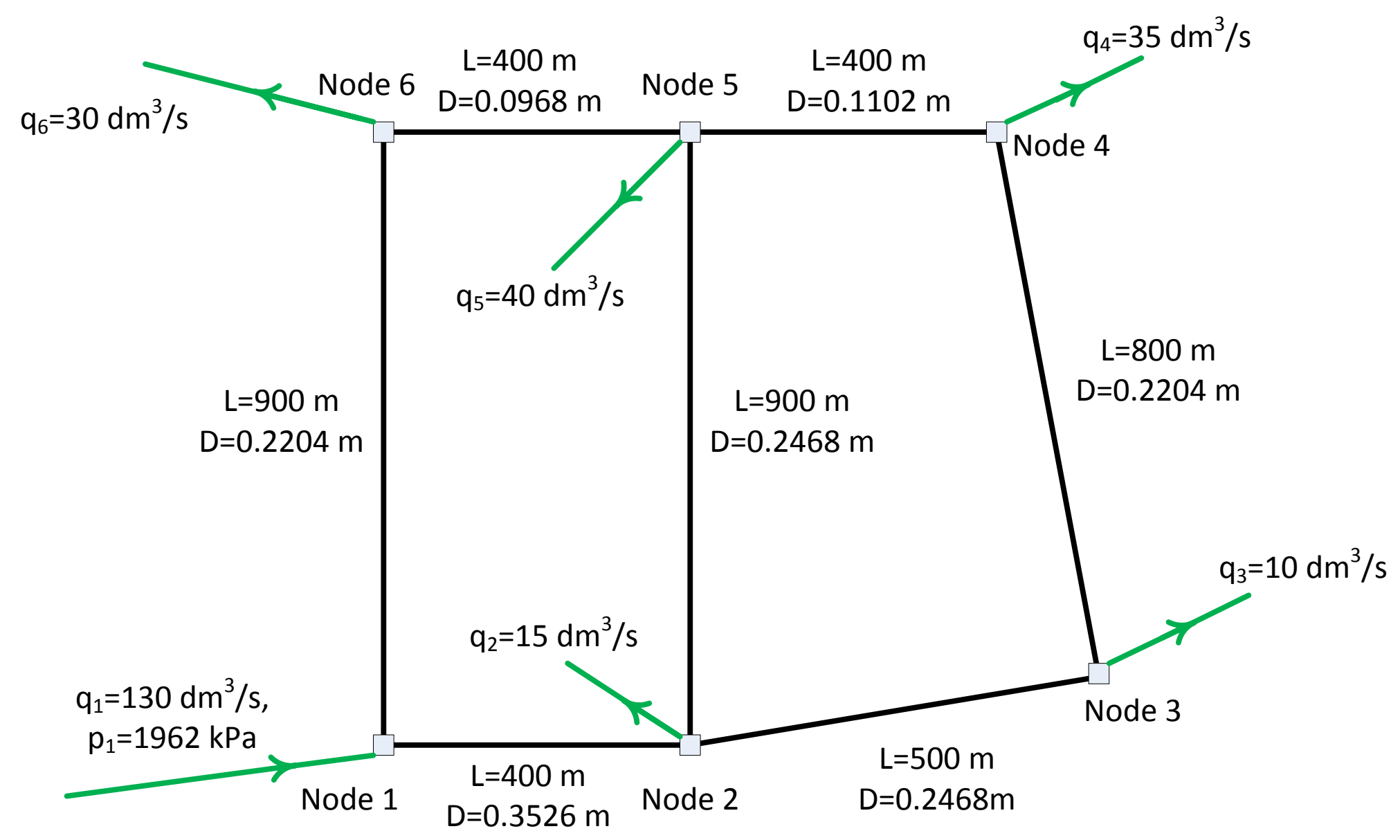

L-length

D-diameter

q-water demands and input, Q-flows

$\mathrm{p}$-pressure, $\Delta \mathrm{p}$-pressure drop

$\varepsilon$-roughness of pipes; $\varepsilon=3 \cdot 10^{-6} \mathrm{~m}$

$v$-kinematic viscosity of water; $v=1.004 \cdot 10^{-6} \mathrm{~m}^{2} / \mathrm{s}$

$\rho$-water density; $\rho=1000 \mathrm{~kg} / \mathrm{m}^{3}$

\section{Final pattern of pressure (after 12 iterations)}

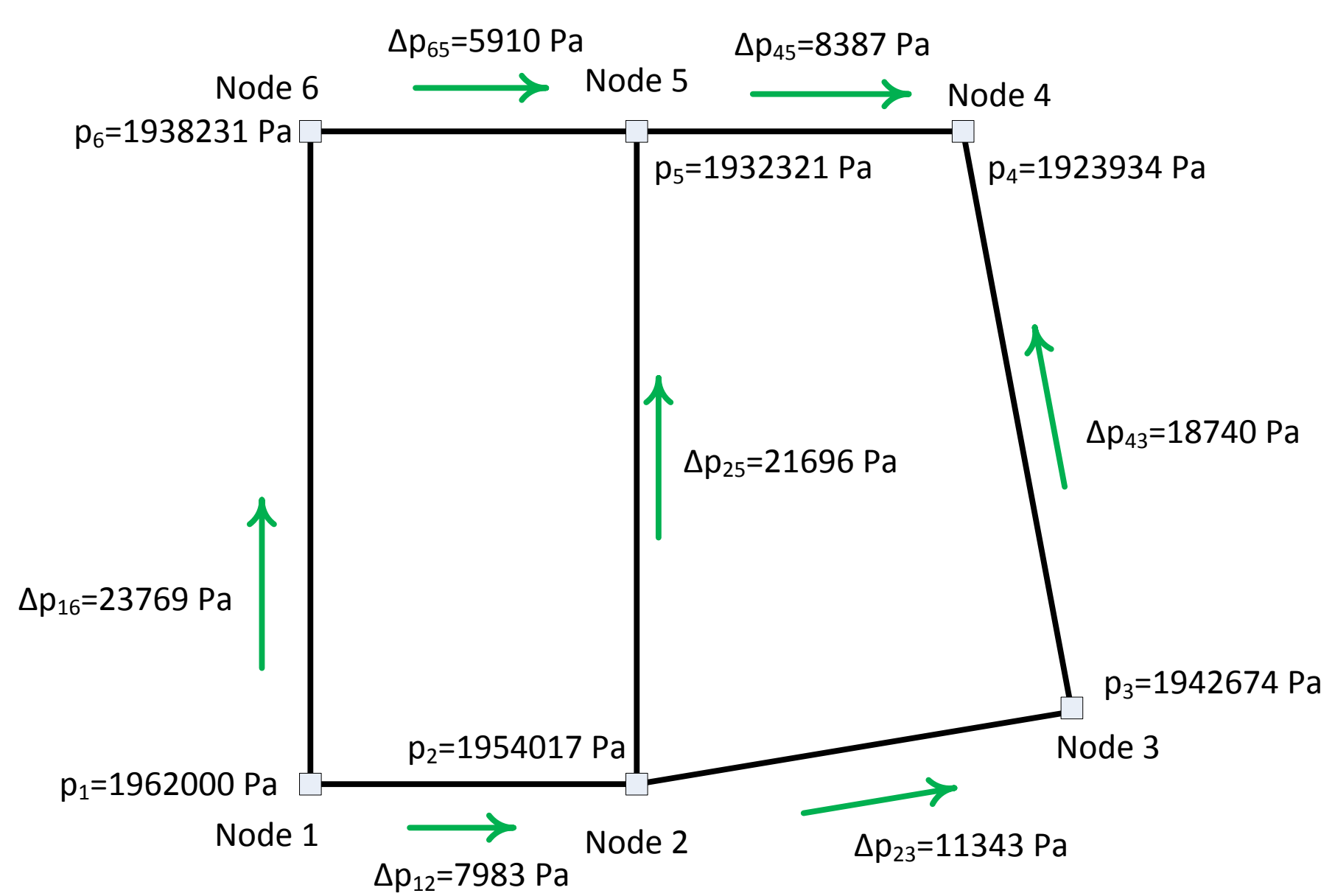

Initial pattern of pressure drops

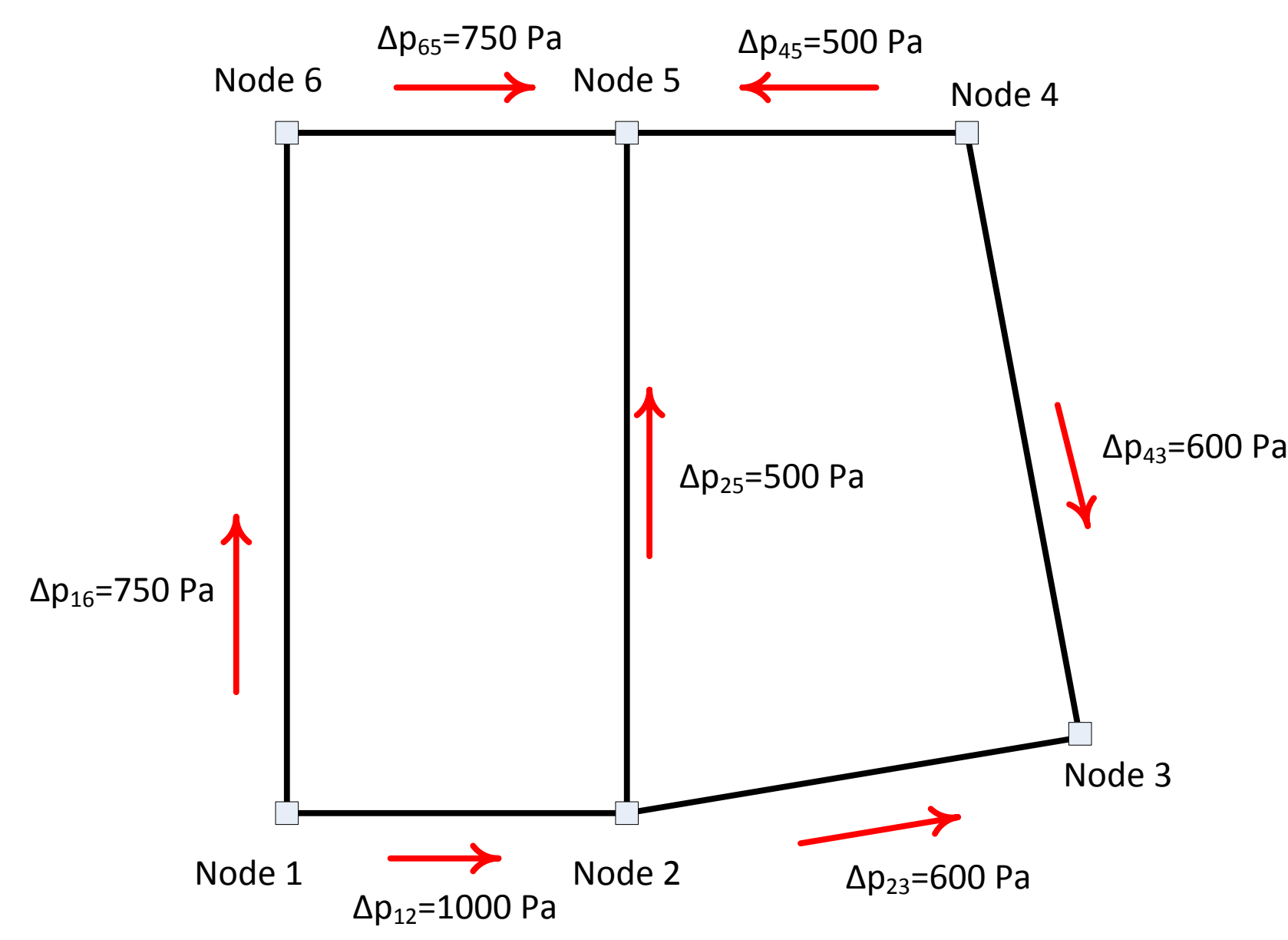

Final flows (after 12 iterations)

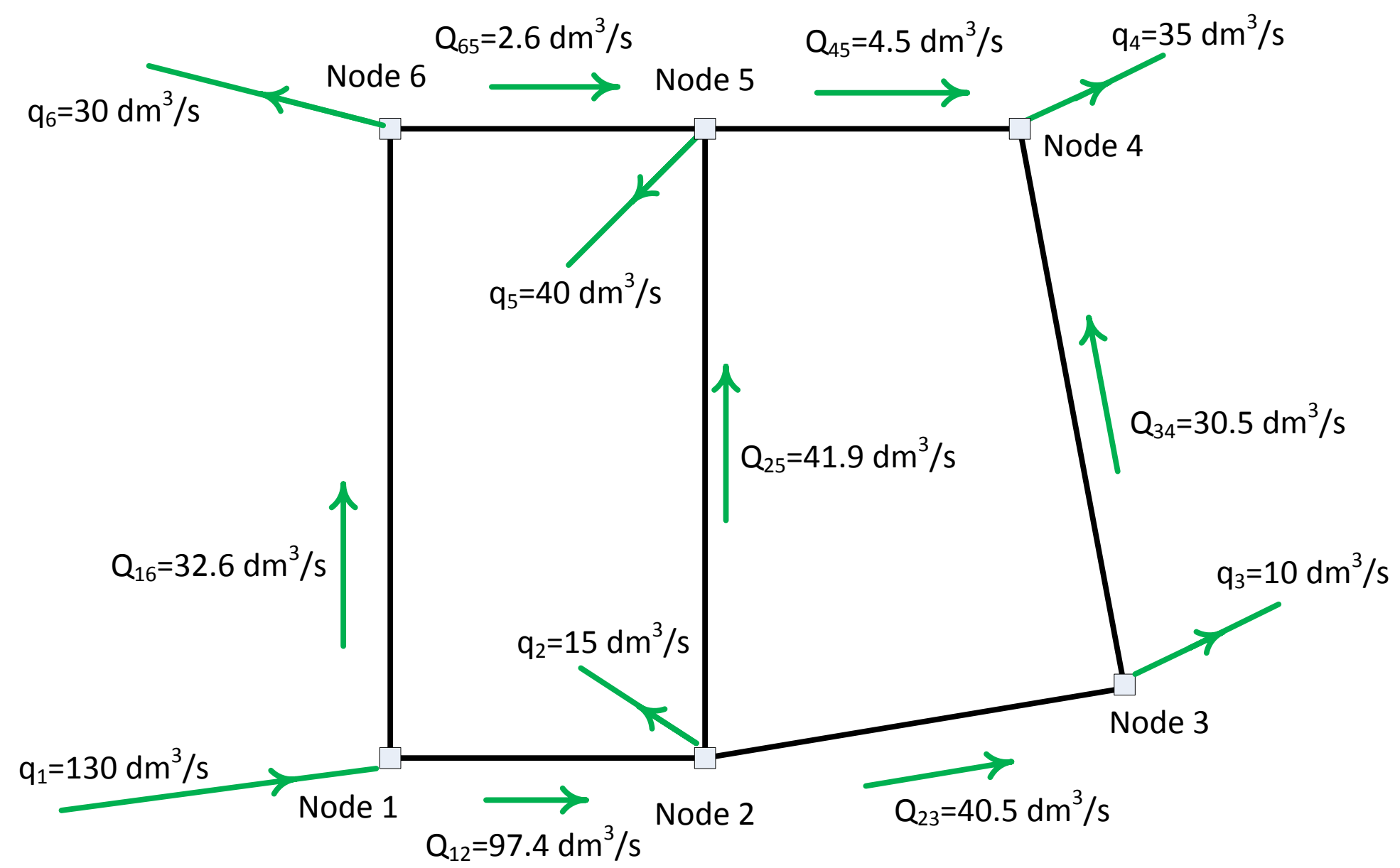




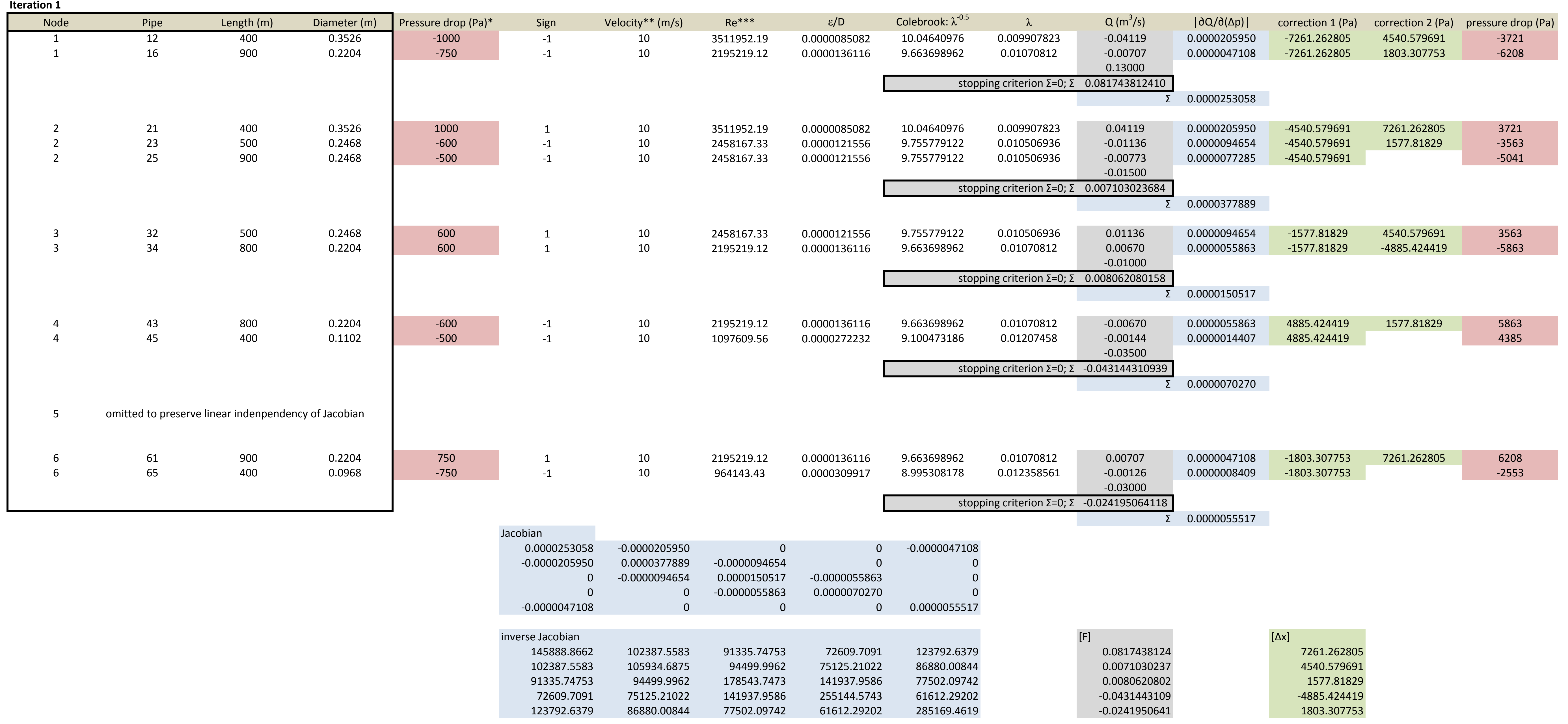




\begin{tabular}{|c|c|c|c|c|c|c|c|c|c|c|c|c|c|c|c|}
\hline Node & Pipe & Length $(\mathrm{m})$ & Diameter $(\mathrm{m})$ & Pressure drop $(\mathrm{Pa})^{*}$ & Sign & Velocity** $(\mathrm{m} / \mathrm{s})$ & $\mathrm{Re}$ & $\varepsilon / D$ & Colebrook: $:^{-0.5}$ & $\lambda$ & $Q\left(\mathrm{~m}^{3} / \mathrm{s}\right)$ & $|\partial Q / \partial(\Delta p)|$ & correction $1(\mathrm{~Pa})$ & correction 2 ( $\mathrm{Pa})$ & pressure drop (Pa) \\
\hline & 12 & 400 & & -3721 & -1 & & & & \multirow{3}{*}{$\begin{array}{l}7.748505571 \\
6.752406402\end{array}$} & 0.016655746 & -0.06128 & 0.0000082349 & -19290.31926 & 14899.69072 & plessure -8111 (pa) \\
\hline \multirow{3}{*}{$\begin{array}{l}1 \\
1\end{array}$} & \multirow{3}{*}{16} & \multirow[t]{3}{*}{900} & \multirow{3}{*}{0.2204} & -6208 & \multirow{3}{*}{-1} & \multirow{3}{*}{0.19} & \multirow{3}{*}{$\begin{array}{l}140654.54 \\
4065.51\end{array}$} & \multirow{3}{*}{0.0000136116} & & 0.021932233 & -0.01421 & 0.0000011441 & -19290.31926 & 3243.047121 & -22255 \\
\hline & & & & & & & & & \multirow{2}{*}{\multicolumn{3}{|c|}{ stopping criterion $\Sigma=0 ; \Sigma \quad 0.054516105450$}} & \multirow{2}{*}{\multicolumn{2}{|c|}{0.0000093790}} & & \\
\hline & & & & & & & & & & & & & & & \\
\hline 2 & 21 & 400 & 0.3526 & 3721 & 1 & 0.42 & 148144.54 & 0.0000085082 & 7.748505571 & 0.016655746 & 0.06128 & 0.0000082349 & -14899.69072 & 19290.31926 & 8111 \\
\hline 2 & 23 & 500 & \multirow{5}{*}{0.2468} & -3563 & -1 & 0.24 & 58364.88 & & \multirow{3}{*}{$\begin{array}{l}7.029694664 \\
6.735132125\end{array}$} & 0.020236112 & -0.01994 & \multirow{3}{*}{$\begin{array}{l}0.00000027990 \\
0.0000016804\end{array}$} & -14899.69072 & 7571.140532 & -10891 \\
\hline \multirow{4}{*}{2} & \multirow{4}{*}{25} & \multirow{4}{*}{900} & & -5041 & \multirow{4}{*}{-1} & \multirow{4}{*}{0.16} & \multirow{4}{*}{39712.27} & \multirow{4}{*}{0.0000121556} & & 0.022044881 & -0.01694 & & \multirow{4}{*}{-14899.69072} & & -19940 \\
\hline & & & & & & & & & & & $\begin{array}{c}-0.01500 \\
\end{array}$ & & & & \\
\hline & & & & & & & & & stop: & g criterion $\Sigma=0 ; \Sigma$ & 0.009393981992 & & & & \\
\hline & & & & & & & & & & & & 0.0000127143 & & & \\
\hline 3 & 32 & 500 & 0.2468 & 3563 & 1 & 0.24 & 58364.88 & 0.0000121556 & 7.029694664 & 0.020236112 & 0.01994 & 0.0000027990 & -7571.140532 & 14899.69072 & 10891 \\
\hline 3 & 34 & 800 & 0.2204 & -5863 & -1 & 0.18 & 38572.05 & 0.0000136116 & 6.71218502 & 0.022195869 & -0.01456 & 0.0000012412 & -7571.140532 & -5239.473291 & -18674 \\
\hline & & & & & & & & & & & -0.01000 & & & & \\
\hline & & & & & & & & & stopt & g criterion $\Sigma=0 ; \Sigma$ & -0.004611303413 & & & & \\
\hline & & & & & & & & & & & & 0.0000040402 & & & \\
\hline 4 & 43 & 800 & 0.2204 & 5863 & 1 & 0.18 & 38572.05 & 0.0000136116 & 6.71218502 & 0.022195869 & 0.01456 & 0.0000012412 & 5239.473291 & 7571.140532 & 18674 \\
\hline 4 & 45 & 400 & 0.1102 & 4385 & 1 & 0.15 & 16579.55 & 0.0000272232 & 6.066929703 & 0.027168276 & 0.00284 & 0.0000003243 & 5239.473291 & & 9625 \\
\hline & & & & & & & & & stopp & g criterion $\Sigma=0 ; \Sigma$ & $\begin{array}{c}-0.03500 \\
-0.017600196387\end{array}$ & & & & \\
\hline & & & & & & & & & & & & 0.0000015655 & & & \\
\hline 5 & omitted to $\mathrm{p}$ & ear indenpen & of Jacobian & & & & & & & & & & & & \\
\hline 6 & 61 & 900 & 0.2204 & 6208 & 1 & 0.19 & 40658.51 & 0.0000136116 & 6.752406402 & 0.021932233 & 0.01421 & 0.0000011441 & -3243.047121 & 19290.31926 & 22255 \\
\hline 6 & 65 & 400 & 0.0968 & -2553 & -1 & 0.17 & 16523.84 & 0.0000309917 & 6.063556479 & 0.027198512 & -0.00157 & 0.0000003072 & -3243.047121 & & -5796 \\
\hline & & & & & & & & & stopt & g criterion $\Sigma=0 ; \Sigma$ & $\frac{-0.03000}{-0.017363570708}$ & & & & \\
\hline & & & & & & & & & & & & 0.0000014513 & & & \\
\hline & & & & & Jacobian & & & & & & & & & & \\
\hline & & & & & 0.0000093790 & -0.0000082349 & 0 & 0 & -0.0000011441 & & & & & & \\
\hline & & & & & -0.0000082349 & 0.0000127143 & -0.0000027990 & 0 & 0 & & & & & & \\
\hline & & & & & & -0.0000027990 & 0.0000040402 & -0.0000012412 & 0 & & & & & & \\
\hline & & & & & & & -0.0000012412 & 0.0000015655 & 0 & & & & & & \\
\hline & & & & & -0.0000011441 & 0 & 0 & 0 & 0.0000014513 & & & & & & \\
\hline & & & & & inverse Jacobian & & & & & & [F] & & {$[\Delta x]$} & & \\
\hline & & & & & 556645.4698 & 451580.4812 & 413586.0364 & 327909.4576 & 438822.2534 & & 0.0545161055 & & 19290.31926 & & \\
\hline & & & & & 451580.4812 & 464860.4479 & 425748.6719 & 337552.5376 & 355996.0066 & & 0.0093939820 & & 14899.69072 & & \\
\hline & & & & & 413586.0364 & 425748.6719 & 717143.8627 & 568583.6425 & 326043.7142 & & -0.0046113034 & & 7571.140532 & & \\
\hline & & & & & 327909.4576 & 337552.5376 & 568583.6425 & 1089553.347 & 258501.9998 & & -0.0176001964 & & -5239.473291 & & \\
\hline & & & & & 438822.2534 & 355996.0066 & 326043.7142 & 258501.9998 & 1034976.103 & & -0.0173635707 & & 3243.047121 & & \\
\hline
\end{tabular}

"from the previous iteration
$* *$ velocity calculated using calculated flow, Q from the previous iteration 
Standard procedure (pressure drop independent Jacobian); Re=Re(O)

\begin{tabular}{|c|c|c|c|c|c|c|c|c|c|c|c|c|c|c|c|}
\hline Node & Pipe & Length $(\mathrm{m})$ & Diameter $(\mathrm{m})$ & Pressure drop $(\mathrm{Pa})^{*}$ & Sign & Velocity (m/s) & $\operatorname{Re}$ & $\varepsilon / \mathrm{D}$ & Colebrook: $\lambda^{-0.5}$ & $\lambda$ & $Q\left(\mathrm{~m}^{3} / \mathrm{s}\right)$ & $|\partial Q / \partial(\Delta p)|$ & correction $1(\mathrm{~Pa})$ & correction $2(\mathrm{~Pa})$ & pressure drop $(\mathrm{Pa})$ \\
\hline \multirow{4}{*}{1} & 12 & 400 & 0.3526 & -8111 & \multirow{4}{*}{-1} & 0.63 & 220396.17 & 0.0000085082 & 8.053574983 & 0.015417806 & -0.09404 & 0.0000057969 & -6197.128544 & 5614.524376 & -8694 \\
\hline & \multirow[t]{3}{*}{16} & \multirow{3}{*}{900} & \multirow{3}{*}{0.2204} & -22255 & & \multirow{3}{*}{0.37} & \multirow{3}{*}{81735.41} & \multirow{3}{*}{0.0000136116} & 7.286294946 & 0.018835906 & -0.02902 & 0.0000006520 & -6197.128544 & 737.810175 & -27715 \\
\hline & & & & & & & & & \multirow{2}{*}{\multicolumn{4}{|c|}{$\begin{array}{llll}\text { stopping criterion } \Sigma=0 ; \Sigma & 0.006936969187 & \\
\Sigma & 0.0000064489\end{array}$}} & & & \\
\hline & & & & & & & & & & & & & & & \\
\hline 2 & 21 & 400 & 0.3526 & 8111 & 1 & 0.63 & 220396.17 & 0.0000085082 & 8.053574983 & 0.015417806 & 0.09404 & 0.0000057969 & -5614.524376 & 6197.128544 & 8694 \\
\hline 2 & 23 & 500 & 0.2468 & -10891 & -1 & 0.42 & 102481.12 & 0.0000121556 & 7.460882598 & 0.017964684 & -0.03701 & 0.0000016990 & -5614.524376 & 3530.014773 & -12976 \\
\hline \multirow[t]{3}{*}{2} & \multirow[t]{3}{*}{25} & \multirow{3}{*}{900} & \multirow{3}{*}{0.2468} & -19940 & \multirow{3}{*}{-1} & \multirow{3}{*}{0.35} & \multirow{3}{*}{87049.07} & \multirow{3}{*}{0.0000121556} & 7.335888597 & 0.018582089 & -0.03670 & 0.0000009202 & -5614.524376 & & -25555 \\
\hline & & & & & & & & & stopp & $\mathrm{g}$ criterion $\Sigma=0 ; \Sigma$ & $\begin{array}{c}-0.01500 \\
0.005331117729\end{array}$ & & & & \\
\hline & & & & & & & & & & & & 0.0000084162 & & & \\
\hline 3 & \multirow{4}{*}{$\begin{array}{l}32 \\
34\end{array}$} & 500 & 0.2468 & 10891 & \multirow{4}{*}{$\begin{array}{c}1 \\
-1\end{array}$} & \multirow{4}{*}{0.38} & 102481.12 & 0.0000121556 & 7.460882598 & 0.017964684 & 0.03701 & 0.0000016990 & -3530.014773 & 5614.524376 & 12976 \\
\hline \multirow[t]{3}{*}{3} & & \multirow{3}{*}{800} & \multirow{3}{*}{0.2204} & -18674 & & & 83750.33 & 0.0000136116 & 7.304920399 & 0.018739976 & -0.02827 & 0.0000007569 & -3530.014773 & 515.961554 & -21688 \\
\hline & & & & & & & & & 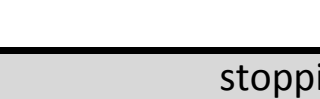 & g criterion $\Sigma=0 ; \Sigma$ & $\begin{array}{c}-0.01000 \\
-0.001260223488\end{array}$ & & & & \\
\hline & & & & & & & & & & & & 0.0000024560 & & & \\
\hline 4 & 43 & 800 & 0.2204 & 18674 & 1 & 0.38 & 83750.33 & 0.0000136116 & 7.304920399 & 0.018739976 & 0.02827 & 0.0000007569 & -515.961554 & 3530.014773 & 21688 \\
\hline 4 & 45 & 400 & 0.1102 & 9625 & 1 & 0.30 & 32733.95 & 0.0000272232 & 6.581436002 & 0.023086531 & 0.00457 & 0.0000002375 & -515.961554 & & 9109 \\
\hline & & & & & & & & & $\begin{array}{l}\text { stopp } \\
\end{array}$ & $\mathrm{g}$ criterion $\Sigma=0 ; \Sigma$ & $\begin{array}{c}-0.03500 \\
-0.002158906865\end{array}$ & & & & \\
\hline & & & & & & & & & & & & 0.0000009944 & & & \\
\hline 5 & omitted to $\mathrm{F}$ & near indenpe & $y$ of Jacobian & & & & & & & & & & & & \\
\hline 6 & 61 & 900 & 0.2204 & 22255 & 1 & 0.37 & 81735.41 & 0.0000136116 & 7.286294946 & 0.018835906 & 0.02902 & 0.0000006520 & -737.810175 & 6197.128544 & 27715 \\
\hline 6 & 65 & 400 & 0.0968 & -5796 & -1 & 0.21 & 20551.49 & 0.0000309917 & 6.228113731 & 0.025780239 & -0.00243 & 0.0000002094 & -737.810175 & & -6534 \\
\hline & & & & & & & & & & & -0.03000 & & & & \\
\hline & & & & & & & & & stopp & $g$ criterion $\Sigma=0 ; \Sigma$ & -0.003405176411 & & & & \\
\hline & & & & & & & & & & & & 0.0000008615 & & & \\
\hline & & & & & Jacobian & & & & & & & & & & \\
\hline & & & & & 0.0000064489 & -0.0000057969 & & 0 & -0.0000006520 & & & & & & \\
\hline & & & & & -0.0000057969 & 0.0000084162 & -0.0000016990 & 0 & 0 & & & & & & \\
\hline & & & & & 0 & -0.0000016990 & 0.0000024560 & -0.0000007569 & 0 & & & & & & \\
\hline & & & & & & 0 & -0.0000007569 & 0.0000009944 & & & & & & & \\
\hline & & & & & -0.0000006520 & 0 & 0 & 0 & 0.0000008615 & & & & & & \\
\hline & & & & & inverse Jacobian & & & & & & F] & & {$[\Delta x]$} & & \\
\hline & & & & & 933294.7716 & 786307.8105 & 710695.2482 & 540972.7189 & 706413.7612 & & 0.0069369692 & & 6197.128544 & & \\
\hline & & & & & 786307.8105 & 807808.4521 & 730128.3552 & 555764.9674 & 595158.8659 & & 0.0053311177 & & 5614.524376 & & \\
\hline & & & & & 710695.2482 & 730128.3552 & 1191889.071 & 907251.6987 & 537927.4787 & & -0.0012602235 & & 3530.014773 & & \\
\hline & & & & & 540972.7189 & 555764.9674 & 907251.6987 & 1696210.772 & 409463.96 & & -0.0021589069 & & 515.961554 & & \\
\hline & & & & & 706413.7612 & 595158.8659 & 537927.4787 & 409463.96 & 1695512.422 & & -0.0034051764 & & 737.810175 & & \\
\hline
\end{tabular}

*from the previous iteration

Dejan Brkic, Strumicka 88, 11050 Belgrade, Serbia; dejanrg@@tesla.rcub.bg.ac.rs, dejanbrkic0611@gmail.com 
Standard procedure (pressure drop independent Jacobian); Re=Re(O)

\begin{tabular}{|c|c|c|c|c|c|c|c|c|c|c|c|c|c|c|c|}
\hline Node & Pipe & Length $(\mathrm{m})$ & Diameter $(\mathrm{m})$ & Pressure drop $(\mathrm{Pa})^{*}$ & Sign & Velocity $(\mathrm{m} / \mathrm{s})$ & $\mathrm{Re}$ & $\varepsilon / \mathrm{D}$ & Colebrook: $\lambda^{-0.5}$ & $\lambda$ & $Q\left(\mathrm{~m}^{3} / \mathrm{s}\right)$ & $|\partial Q / \partial(\Delta p)|$ & correction $1(\mathrm{~Pa})$ & correction $2(\mathrm{~Pa})$ & pressure drop $(\mathrm{Pa})$ \\
\hline 1 & 12 & 400 & 0.3526 & -8694 & \multirow{4}{*}{-1} & 0.96 & 338227.57 & 0.0000085082 & 8.380949593 & 0.014236838 & -0.10132 & 0.0000058269 & 4093.117544 & \multirow{4}{*}{$\begin{array}{l}-5621.522911 \\
-566.7642687\end{array}$} & \multirow{4}{*}{$\begin{array}{l}-8022 \\
-24188\end{array}$} \\
\hline \multirow{3}{*}{1} & \multirow[t]{3}{*}{16} & \multirow[t]{3}{*}{900} & \multirow[t]{3}{*}{0.2204} & -27715 & & \multirow[t]{3}{*}{0.76} & \multirow[t]{3}{*}{166993.74} & \multirow[t]{3}{*}{0.0000136116} & 7.831619812 & 0.016304099 & -0.03481 & \multirow[t]{2}{*}{0.0000006280} & \multirow[t]{3}{*}{4093.117544} & & \\
\hline & & & & & & & & & \multirow{2}{*}{\multicolumn{2}{|c|}{ stopping criterion $\Sigma=0 ; \Sigma$}} & $\begin{array}{c}0.13000 \\
-0006127957496\end{array}$ & & & & \\
\hline & & & & & & & & & & & & 0.0000064549 & & & \\
\hline 2 & 21 & 400 & 0.3526 & 8694 & 1 & 0.96 & 338227.57 & 0.0000085082 & 8.380949593 & 0.014236838 & 0.10132 & 0.0000058269 & 3421.522911 & -4093.117544 & 8022 \\
\hline 2 & 23 & 500 & 0.2468 & -12976 & -1 & 0.77 & 190171.01 & 0.0000121556 & 7.933209399 & 0.015889205 & -0.04295 & \multirow{3}{*}{$\begin{array}{l}0.0000016551 \\
0.0000008784\end{array}$} & \multirow{4}{*}{$\begin{array}{l}3421.522911 \\
3421.522911\end{array}$} & -1948.574579 & -11503 \\
\hline \multirow{3}{*}{2} & \multirow[t]{3}{*}{25} & \multirow[t]{3}{*}{900} & \multirow{3}{*}{0.2468} & -25555 & \multirow[t]{3}{*}{-1} & \multirow{3}{*}{0.77} & \multirow{3}{*}{188580.17} & \multirow{3}{*}{0.0000121556} & 7.926813297 & 0.015914857 & -0.04489 & & & & -22133 \\
\hline & & & & & & & & & stoppin & g criterion $\Sigma=0 ; \Sigma$ & $\begin{array}{c}-0.01500 \\
-0.001530003977\end{array}$ & & & & \\
\hline & & & & & & & & & & & & 0.0000083604 & & & \\
\hline 3 & 32 & 500 & 0.2468 & 12976 & 1 & 0.77 & 190171.01 & 0.0000121556 & 7.933209399 & 0.015889205 & 0.04295 & 0.0000016551 & 1948.574579 & -3421.522911 & 11503 \\
\hline 3 & 34 & 800 & 0.2204 & -21688 & -1 & 0.74 & 162662.02 & 0.0000136116 & 7.81162916 & 0.016387653 & -0.03258 & 0.0000007511 & 1948.574579 & 798.7639351 & -18941 \\
\hline & & & & & & & & & stoopin & $g$ criterion $\Sigma=0: \Sigma$ & $\begin{array}{c}-0.01000 \\
0.00037436202\end{array}$ & & & & \\
\hline & & & & & & & & & & & & 0.0000024062 & & & \\
\hline 4 & 43 & 800 & 0.2204 & 21688 & 1 & 0.74 & 162662.02 & 0.0000136116 & 7.81162916 & 0.016387653 & 0.03258 & 0.0000007511 & -798.7639351 & -1948.574579 & 18941 \\
\hline 4 & 45 & 400 & 0.1102 & 9109 & 1 & 0.48 & 52606.86 & 0.0000272232 & 6.940828154 & 0.020757613 & 0.00469 & 0.0000002574 & -798.7639351 & & 8310 \\
\hline & & & & & & & & & stoppin & g criterion $\Sigma=0 ; \Sigma$ & $\begin{array}{c}0.002269000 \\
\end{array}$ & & & & \\
\hline 5 & omitted to $\mathrm{p}$ & inear indenpe & $y$ of Jacobian & & & & & & & & & & & & \\
\hline 6 & 61 & 900 & 0.2204 & 27715 & 1 & 0.76 & 166993.74 & 0.0000136116 & 7.831619812 & 0.016304099 & 0.03481 & 0.0000006280 & 566.7642687 & -4093.117544 & 24188 \\
\hline 6 & 65 & 400 & 0.0968 & -6534 & -1 & 0.33 & 31805.22 & 0.0000309917 & 6.558142742 & 0.02325082 & -0.00271 & 0.0000002077 & 566.7642687 & & -5967 \\
\hline & & & & & & & & & stoppin & g criterion $\Sigma=0 ; \Sigma$ & $\begin{array}{c}-0.03000 \\
0.002096943068\end{array}$ & & & & \\
\hline & & & & & & & & & & & & 0.0000008357 & & & \\
\hline & & & & & Jacobian & & & & & & & & & & \\
\hline & & & & & 0.0000064549 & -0.0000058269 & 0 & 0 & -0.0000006280 & & & & & & \\
\hline & & & & & -0.0000058269 & 0.0000083604 & -0.0000016551 & 0 & 0 & & & & & & \\
\hline & & & & & & -0.0000016551 & 0.0000024062 & -0.0000007511 & 0 & & & & & & \\
\hline & & & & & & & -0.0000007511 & 0.0000010085 & 0 & & & & & & \\
\hline & & & & & -0.0000006280 & 0 & 0 & 0 & 0.0000008357 & & & & & & \\
\hline & & & & & inverse Jacobian & & & & & & [F] & & & & \\
\hline & & & & & 956117.2803 & 810109.0778 & 726010.6185 & 540687.0449 & 718505.1279 & & -0.0061279575 & & -4093.117544 & & \\
\hline & & & & & 810109.0778 & 831808.3481 & 745457.2598 & 555169.6802 & 608782.5611 & & -0.0015300040 & & -3421.522911 & & \\
\hline & & & & & 726010.6185 & 745457.2598 & 1209528.934 & 900781.0746 & 545584.0649 & & 0.0003744362 & & $\begin{array}{r}-1948.574579 \\
\end{array}$ & & \\
\hline & & & & & 540687.0449 & 555169.6802 & 900781.0746 & 1662387.64 & 406316.696 & & 0.0022691308 & & 798.7639351 & & \\
\hline & & & & & 718505.1279 & 608782.5611 & 545584.0649 & 406316.696 & 1736514.579 & & 0.0020969431 & & -566.7642687 & & \\
\hline
\end{tabular}

*from the previous iteration

Dejan Brkic, Strumicka 88, 11050 Belgrade, Serbia; dejanrgf@tesla.rcub.bg.ac.rs, dejanbrkic0611@gmail.com 


\begin{tabular}{|c|c|c|c|c|c|c|c|c|c|c|c|c|c|c|c|}
\hline Node & Pipe & Length $(m)$ & Diameter $(\mathrm{m})$ & Pressure drop (Pa)* & Sign & Velocity $(\mathrm{m} / \mathrm{s})$ & $\operatorname{Re}$ & $\varepsilon / D$ & Colebrook: $\lambda^{-0.5}$ & $\lambda$ & $Q\left(\mathrm{~m}^{3} / \mathrm{s}\right)$ & $|\partial Q / \partial(\Delta p)|$ & correction $1(\mathrm{~Pa})$ & correction $2(\mathrm{~Pa})$ & pressure drop $(\mathrm{Pa})$ \\
\hline \multirow{4}{*}{1} & 12 & 400 & 0.3526 & $\begin{array}{l}-8022 \\
20100\end{array}$ & \multirow{4}{*}{-1} & \multirow{4}{*}{0.91} & \multirow{4}{*}{200300.90} & \multirow{4}{*}{0.0000136116} & 8.437656496 & 0.014046118 & -0.09798 & 0.0000061069 & 822.7871268 & -726.6128476 & $\begin{array}{l}-7926 \\
72071\end{array}$ \\
\hline & \multirow[t]{3}{*}{16} & \multirow[t]{3}{*}{900} & \multirow[t]{3}{*}{0.2204} & -24188 & & & & & 7.969712731 & 0.015743985 & -0.03309 & 0.0000006841 & 822.7871268 & -105.5573975 & -23471 \\
\hline & & & & & & & & & \multirow{2}{*}{\multicolumn{4}{|c|}{$\begin{array}{cccc}\text { stopping criterion } \Sigma=0 ; \Sigma & -0.001077990309 & \\
\Sigma & 0.0000067910\end{array}$}} & & & \\
\hline & & & & & & & & & & & & & & & \\
\hline 2 & 21 & 400 & 0.3526 & 8022 & 1 & 1.04 & 364397.76 & 0.0000085082 & 8.437656496 & 0.014046118 & 0.09798 & 0.0000061069 & 726.6128476 & -822.7871268 & 7926 \\
\hline 2 & 23 & 500 & 0.2468 & -11503 & -1 & 0.90 & 220714.24 & \multirow{4}{*}{$\begin{array}{l}0.0000121556 \\
0.0000121556\end{array}$} & \multirow{3}{*}{$\begin{array}{l}8.046465161 \\
8.079990194\end{array}$} & 0.015445065 & -0.04102 & 0.0000017830 & 726.6128476 & -439.5999529 & -11216 \\
\hline \multirow{3}{*}{2} & \multirow{3}{*}{25} & \multirow{3}{*}{900} & \multirow{3}{*}{0.2468} & -22133 & \multirow{3}{*}{-1} & \multirow{3}{*}{0.94} & 230681.41 & & & 0.015317163 & -0.04259 & 0.0000009621 & 726.6128476 & 0 & -21407 \\
\hline & & & & & & & & & \multirow{2}{*}{\multicolumn{3}{|c|}{ stopping criterion $\Sigma=0 ; \Sigma \quad-0.000623468032$}} & & & & \\
\hline & & & & & & & & & & & & 0.0000088520 & & & \\
\hline 3 & 32 & 500 & 0.2468 & 11503 & \multirow{4}{*}{$\begin{array}{c}1 \\
-1\end{array}$} & 0.90 & 220714.24 & 0.0000121556 & 8.046465161 & 0.015445065 & 0.04102 & 0.0000017830 & 439.5999529 & -726.6128476 & 11216 \\
\hline \multirow[t]{3}{*}{3} & \multirow[t]{3}{*}{34} & \multirow[t]{3}{*}{800} & \multirow[t]{3}{*}{0.2204} & -18941 & & \multirow[t]{3}{*}{0.85} & 187458.07 & 0.0000136116 & 7.919447902 & 0.015944474 & -0.03087 & 0.0000008148 & 439.5999529 & -0.015372369 & -18501 \\
\hline & & & & & & & & & stopp & g criterion $\Sigma=0 ; \varepsilon$ & |-0.01500 & & & & \\
\hline & & & & & & & & & s & & $\Sigma$ & 0.0000025978 & & & \\
\hline 4 & 43 & 800 & 0.2204 & 18941 & 1 & 0.85 & 187458.07 & 0.0000136116 & 7.919447902 & 0.015944474 & 0.03087 & 0.0000008148 & 0.015372369 & -439.5999529 & 18501 \\
\hline 4 & 45 & 400 & 0.1102 & 8310 & 1 & 0.49 & 53972.04 & 0.0000272232 & 6.960221573 & 0.0206421 & 0.00449 & 0.0000002703 & 0.015372369 & & 8310 \\
\hline & & & & & & & & & stopp & g criterion $\Sigma=0 ; \varepsilon$ & 0.000358174487 & & & & \\
\hline & & & & & & & & & & & & 0.0000010851 & & & \\
\hline 5 & omitted to $\mathrm{F}$ & ear indenper & y of Jacobian & & & & & & & & & & & & \\
\hline 6 & 61 & 900 & 0.2204 & 24188 & 1 & 0.91 & 200300.90 & 0.0000136116 & 7.969712731 & 0.015743985 & & 0.0000006841 & 105.56 & -823 & 23471 \\
\hline 6 & 65 & 400 & 0.0968 & -5967 & -1 & 0.37 & 35558.24 & 0.0000309917 & 6.642491483 & 0.022664074 & -0.00263 & 0.0000002201 & 105.56 & & -5862 \\
\hline & & & & & & & & & stopp & g criterion $\Sigma=0 ; \varepsilon$ & $\begin{array}{c}-0.004600 \\
0.000467254\end{array}$ & & & & \\
\hline & & & & & & & & & & & & 0.0000009042 & & & \\
\hline & & & & & Jacobian & & & & & & & & & & \\
\hline & & & & & 0.0000067910 & -0.0000061069 & 0 & 0 & -0.0000006841 & & & & & & \\
\hline & & & & & -0.0000061069 & 0.0000088520 & -0.0000017830 & 0 & & & & & & & \\
\hline & & & & & 0 & -0.0000017830 & 0.0000025978 & -0.0000008148 & 0 & & & & & & \\
\hline & & & & & & & -0.0000008148 & 0.0000010851 & 0 & & & & & & \\
\hline & & & & & -0.0000006841 & 0 & 0 & 0 & 0.0000009042 & & & & & & \\
\hline & & & & & inverse Jacobian & & & & & & {$[F]$} & & {$[\Delta x]$} & & \\
\hline & & & & & 884762.7501 & 745142.0769 & 668991.5563 & 502354.9599 & 669376.2731 & & -0.0010779903 & & -822.7871268 & & \\
\hline & & & & & 745142.0769 & 765462.4548 & 687235.2734 & 516054.4179 & 563744.8303 & & -0.0006234680 & & -726.6128476 & & \\
\hline & & & & & 668991.5563 & 687235.2734 & 1120534.279 & 841424.6002 & 506132.3781 & & 0.0001535694 & & -439.5999529 & & \\
\hline & & & & & 502354.9599 & 516054.4179 & 841424.6002 & 1553416.841 & 380061.7632 & & 0.0003581745 & & -0.015372369 & & \\
\hline & & & & & 669376.2731 & 563744.8303 & 506132.3781 & 380061.7632 & 1612333.78 & & 0.0004674254 & & -105.5573975 & & \\
\hline
\end{tabular}

${ }^{*}$ from the previous iteration

Dejeration

(a) 
Standard procedure (pressure drop independent Jacobian); Re=Re(Q)
Iteration 6

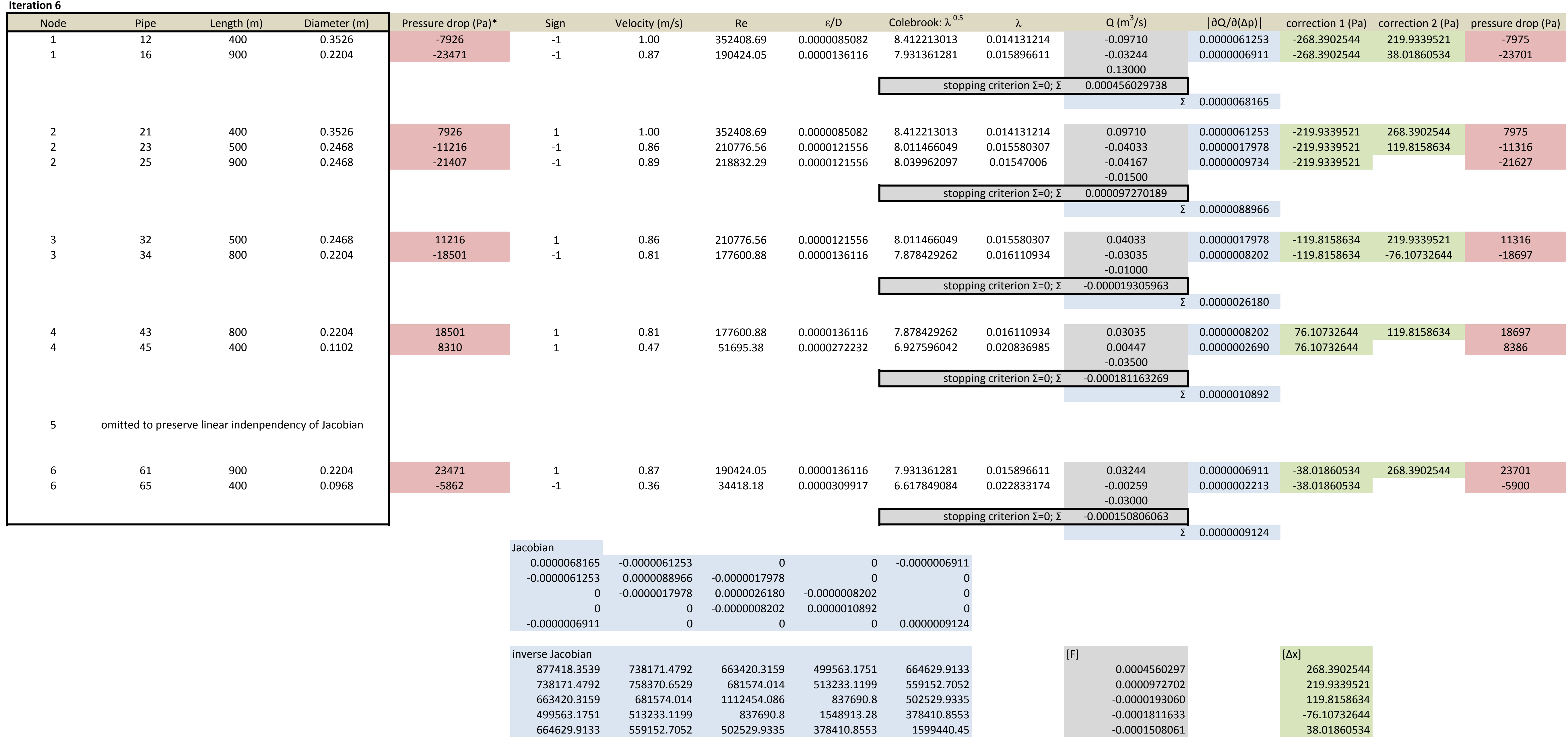

*from the previous iteration

Dejan Brkic, Strumicka 88, 11050 Belgrade, Serbia; dejanrgf@etesla.rcub.bg.ac.rs, dejanbrkkic0611@gmail.com 


\begin{tabular}{|c|c|c|c|c|c|c|c|c|c|c|c|c|c|c|c|}
\hline Node & Pipe & Length $(\mathrm{m})$ & Diameter $(\mathrm{m})$ & Pressure drop $(\mathrm{Pa})^{*}$ & Sign & Velocity (m/s) & $\mathrm{Re}$ & $\varepsilon / D$ & Colebrook: $\lambda^{-0.5}$ & $\lambda$ & $Q\left(\mathrm{~m}^{3} / \mathrm{s}\right)$ & $|\partial Q / \partial(\Delta p)|$ & correction 1 (Pa) & correction 2 (Pa) & pressure drop $(\mathrm{Pa})$ \\
\hline \multirow{4}{*}{$\begin{array}{l}1 \\
1\end{array}$} & 12 & 400 & 0.3526 & -7975 & -1 & 0.99 & 349233.64 & 0.0000085082 & 8.40532649 & 0.014154379 & -0.09732 & 0.0000061017 & -103.2760659 & 90.00523078 & \multirow{4}{*}{$\begin{array}{l}-7988 \\
-23791\end{array}$} \\
\hline & \multirow[t]{3}{*}{16} & \multirow[t]{3}{*}{900} & \multirow[t]{3}{*}{0.2204} & -23701 & \multirow[t]{3}{*}{-1} & \multirow[t]{3}{*}{0.85} & \multirow[t]{3}{*}{186676.91} & \multirow[t]{3}{*}{0.0000136116} & 7.916278277 & 0.015957244 & $\begin{array}{l}-0.03254 \\
0.13000\end{array}$ & \multirow[t]{2}{*}{0.0000006865} & \multirow[t]{3}{*}{-103.2760659} & 13.54338157 & \\
\hline & & & & & & & & & \multirow{2}{*}{\multicolumn{3}{|c|}{$\begin{array}{ll}\text { stopping criterion } \Sigma=0 ; \Sigma \quad 0.000142572813 \\
\end{array}$}} & & & & \\
\hline & & & & & & & & & & & & 0.0000067882 & & & \\
\hline 2 & 21 & 400 & 0.3526 & 7975 & 1 & 0.99 & 349233.64 & 0.0000085082 & 8.40532649 & 0.014154379 & 0.09732 & 0.0000061017 & -90.00523078 & 103.2760659 & 7988 \\
\hline 2 & 23 & 500 & 0.2468 & -11316 & -1 & 0.84 & 207225.07 & 0.0000121556 & 7.998549009 & 0.015630669 & -0.04044 & \multirow{4}{*}{$\begin{array}{l}0.0000017870 \\
0.0000009665\end{array}$} & \multirow{4}{*}{$\begin{array}{l}-90.00523078 \\
-90.00523078\end{array}$} & 53.19904573 & -11353 \\
\hline \multirow[t]{3}{*}{2} & \multirow[t]{3}{*}{25} & \multirow[t]{3}{*}{900} & \multirow[t]{3}{*}{0.2468} & -21627 & \multirow[t]{3}{*}{-1} & \multirow[t]{3}{*}{0.87} & \multirow[t]{3}{*}{214144.15} & \multirow{3}{*}{0.0000121556} & 8.023511147 & 0.015533563 & -0.04180 & & & & \multirow{3}{*}{-21717} \\
\hline & & & & & & & & & & & $\begin{array}{c}-0.01500 \\
000077007\end{array}$ & & & & \\
\hline & & & & & & & & & stoppin & g criterion $\Sigma=0 ; \Sigma$ & 0.000071784087 & & & & \\
\hline 3 & 32 & 500 & & 11316 & 1 & 084 & ה ד & & & & & & & & \\
\hline o & 24 & 300 & 0.2468 & 11316 & 1 & 0.04 & $20 / 225.01$ & 0.0000121556 & 1.998549009 & 0.015630669 & 0.04044 & 0.0000017870 & -53.19904573 & 90.00523078 & 11353 \\
\hline 3 & 34 & 800 & 0.2204 & -18697 & -1 & 0.80 & 174618.70 & 0.0000136116 & 7.865562181 & 0.016163688 & -0.03046 & 0.0000008145 & -53.19904573 & -8.565709334 & -18759 \\
\hline & & & & & & & & & stoppin & g criterion $\Sigma=0 ; \Sigma$ & -0.000015462794 & & & & \\
\hline & & & & & & & & & & & & 0.0000026015 & & & \\
\hline 4 & 43 & 800 & 0.2204 & 18697 & 1 & 0.80 & 174618.70 & 0.0000136116 & 7.865562181 & 0.016163688 & 0.03046 & 0.0000008145 & 8.565709334 & 53.19904573 & 18759 \\
\hline 4 & 45 & 400 & 0.1102 & 8386 & 1 & 0.47 & 51453.11 & 0.0000272232 & 6.924039497 & 0.020858397 & 0.00449 & 0.0000002677 & 8.565709334 & & 8395 \\
\hline & & & & & & & & & stoppin & criterion $\Sigma=0 ; \Sigma$ & $\begin{array}{c}-0.03500 \\
-0.000052601500\end{array}$ & & & & \\
\hline & & & & & & & & & & & & 0.0000010822 & & & \\
\hline 5 & omitted to $\mathrm{F}$ & linear indenp & cy of Jacobian & & & & & & & & & & & & \\
\hline 6 & 61 & 900 & 0.2204 & 23701 & 1 & 0.85 & 186676.91 & 0.0000136116 & 7.916278277 & 0.015957244 & 0.03254 & 0.0000006865 & -13.54338157 & 103.2760659 & 23791 \\
\hline 6 & 65 & 400 & 0.0968 & -5900 & -1 & 0.35 & 33985.86 & 0.0000309917 & 6.608290265 & 0.022899277 & -0.00260 & 0.0000002202 & -13.54338157 & & -5913 \\
\hline & & & & & & & & & stoppin & g criterion $\Sigma=0 ; \Sigma$ & $\begin{array}{c}-0.03000 \\
-0.000058615355\end{array}$ & & & & \\
\hline & & & & & & & & & & & & 0.0000009067 & & & \\
\hline & & & & & Jacobian & & & & & & & & & & \\
\hline & & & & & 0.0000067882 & -0.0000061017 & 0 & 0 & -0.0000006865 & & & & & & \\
\hline & & & & & -0.0000061017 & 0.0000088551 & -0.0000017870 & $\begin{array}{r}0 \\
0\end{array}$ & 0 & & & & & & \\
\hline & & & & & & -0.0000017870 & 0.0000026015 & -0.0000008145 & 0 & & & & & & \\
\hline & & & & & & 0 & -0.0000008145 & 0.0000010822 & 0 & & & & & & \\
\hline & & & & & -0.0000006865 & 0 & 0 & 0 & 0.0000009067 & & & & & & \\
\hline & & & & & inverse Jacobian & & & & & & {$[\mathrm{F}]$} & & {$[\Delta x]$} & & \\
\hline & & & & & 882915.2641 & 743154.417 & 667862.6001 & 502680.877 & 668451.4312 & & 0.0001425728 & & 103.2760659 & & \\
\hline & & & & & 743154.417 & 763463.0485 & 686113.6864 & 516417.9422 & 562639.0819 & & 0.0000717841 & & 90.00523078 & & \\
\hline & & & & & 667862.6001 & 686113.6864 & 1119510.719 & 842623.363 & 505635.9642 & & -0.0000154628 & & 53.19904573 & & \\
\hline & & & & & 502680.877 & 516417.9422 & 842623.363 & 1558281.129 & 380577.5767 & & -0.0000526015 & & -8.565709334 & & \\
\hline & & & & & 668451.4312 & 562639.0819 & 505635.9642 & 380577.5767 & 1608975.255 & & -0.0000586154 & & 13.54338157 & & \\
\hline
\end{tabular}

${ }^{*}$ from the previous iteration

Dejan Brkic, Strumicka 88, 11050 Bed flow, Q from the previous iteration

88, 11050 Belgrade, Serbia; dejanrgf@tesla.rcub.bg.ac.rs, dejanbrkic0611@gmail.com 
Standard procedure (pressure drop independent Jacobian); Re=Re(Q)

\begin{tabular}{|c|c|c|c|c|c|c|c|c|c|c|c|c|c|c|c|}
\hline Node & Pipe & Length $(\mathrm{m})$ & Diameter (m) & Pressure drop $(\mathrm{Pa})^{*}$ & Sign & Velocity (m/s) & $\mathrm{Re}$ & $\varepsilon / D$ & Colebrook: $\lambda^{-0.5}$ & $\lambda$ & $\mathrm{Q}\left(\mathrm{m}^{3} / \mathrm{s}\right)$ & $|\partial Q / \partial(\Delta p)|$ & correction $1(\mathrm{~Pa})$ & correction $2(\mathrm{~Pa})$ & pressure drop ( $\mathrm{Pa})$ \\
\hline 1 & 12 & 400 & 0.3526 & -7988 & -1 & 1.00 & 350012.76 & 0.0000085082 & 8.407022272 & 0.01414867 & -0.09742 & 0.0000060979 & 15.64678921 & -12.4224294 & \\
\hline \multirow{3}{*}{$\begin{array}{l}1 \\
1\end{array}$} & \multirow[t]{3}{*}{16} & \multirow[t]{3}{*}{900} & \multirow[t]{3}{*}{0.2204} & -23791 & \multirow[t]{3}{*}{-1} & \multirow[t]{3}{*}{0.85} & \multirow[t]{3}{*}{187234.06} & \multirow[t]{3}{*}{0.0000136116} & 7.918540357 & 0.015948129 & -0.03261 & \multirow[t]{3}{*}{0.0000006854} & \multirow[t]{3}{*}{15.64678921} & \multirow[t]{3}{*}{-2.204883833} & \multirow[t]{3}{*}{-23778} \\
\hline & & & & & & & & & \multirow{2}{*}{\multicolumn{3}{|c|}{$\begin{array}{|ll|}\text { stopping criterion } \Sigma=0 ; \Sigma & -0.000028874273 \\
\end{array}$}} & & & & \\
\hline & & & & & & & & & & & & & & & \\
\hline \multirow{5}{*}{$\begin{array}{l}2 \\
2 \\
2\end{array}$} & 21 & 400 & 0.3526 & 7988 & 1 & 100 & 35001276 & 0.0000085082 & 8.407022272 & 0.01414867 & 0.09742 & & 12.4224294 & $-15,64678921$ & 7985 \\
\hline & \multirow{4}{*}{$\begin{array}{l}23 \\
25\end{array}$} & 500 & 0.2468 & -11353 & -1 & 0.85 & 207812.31 & 0.0000121556 & 8.000700322 & 0.015622265 & -0.04052 & \multirow{3}{*}{$\begin{array}{l}0.0000017846 \\
0.0000009647\end{array}$} & \multirow{4}{*}{$\begin{array}{l}12.4224294 \\
12.4224294\end{array}$} & \multirow{4}{*}{-6.280508448} & \multirow{4}{*}{$\begin{array}{l}-11347 \\
-21704\end{array}$} \\
\hline & & \multirow[t]{3}{*}{900} & \multirow[t]{3}{*}{0.2468} & -21717 & -1 & 0.87 & 214800.99 & 0.0000121556 & 8.025838021 & 0.015524557 & -0.04190 & & & & \\
\hline & & & & & & & & & \multirow{2}{*}{\multicolumn{3}{|c|}{$\begin{array}{|ll|}\text { stopping criterion } \Sigma=0 ; \Sigma & -0.000003283335 \\
\end{array}$}} & & & & \\
\hline & & & & & & & & & & & & 0.0000088472 & & & \\
\hline 3 & 32 & 500 & 0.2468 & 11353 & 1 & 0.85 & 207812.31 & 0.0000121556 & 8.000700322 & 0.015622265 & 0.04052 & 0.0000017846 & 6.280508448 & -12.4224294 & 11347 \\
\hline \multirow[t]{3}{*}{3} & 34 & 800 & 0.2204 & -18759 & -1 & 0.80 & 175254.17 & 0.0000136116 & 7.868322633 & 0.016152349 & -0.03052 & 0.0000008135 & 6.280508448 & 6.976695969 & -18745 \\
\hline & & & & & & & & & stoppin & criterion $\Sigma=0 . \Sigma$ & $\begin{array}{c}-0.01000 \\
0.000000176288\end{array}$ & & & & \\
\hline & & & & & & & & & & & & 0.0000025980 & & & \\
\hline 4 & 43 & 800 & 0.2204 & 18759 & 1 & 0.80 & 175254.17 & 0.0000136116 & 7.868322633 & 0.016152349 & 0.03052 & 0.0000008135 & -6.976695969 & -6.280508448 & 18745 \\
\hline 4 & 45 & 400 & 0.1102 & 8395 & 1 & 0.47 & 51661.65 & 0.0000272232 & 6.927101861 & 0.020839959 & 0.00449 & 0.0000002676 & -6.976695969 & & 8388 \\
\hline & & & & & & & & & stonnin & criterion $\Sigma=0 ; \Sigma$ & $\begin{array}{c}-.03350 \\
0.00012651510\end{array}$ & & & & \\
\hline 5 & omitted to $\mathrm{F}$ & linear inden & cy of Jacobian & & & & & & & & & & & & \\
\hline 6 & 61 & 900 & 0.2204 & 23791 & 1 & 0.85 & 187234.06 & 0.0000136116 & 7.918540357 & 0.015948129 & 0.03261 & 0.0000006854 & 2.204883833 & -15.64678921 & 23778 \\
\hline 6 & 65 & 400 & 0.0968 & -5913 & -1 & 0.35 & 34046.65 & 0.0000309917 & 6.609641599 & 0.022889915 & -0.00260 & 0.0000002200 & 2.204883833 & & -5911 \\
\hline & & & & & & & & & stoppin & criterion $\Sigma=0 ; \Sigma$ & $\begin{array}{c}-0.03000 \\
0.000008727435\end{array}$ & & & & \\
\hline & & & & & & & & & & & & 0.0000009054 & & & \\
\hline & & & & & Jacobian & & & 0 & م 000000соге & & & & & & \\
\hline & & & & & 0.0000067832 & $\begin{array}{r}-0.0000060979 \\
0.000008472\end{array}$ & $\begin{array}{r}0 \\
-0000017846\end{array}$ & 0 & -0.0000006854 & & & & & & \\
\hline & & & & & $\begin{array}{r}-0.0000060979 \\
0\end{array}$ & -0.0000017846 & 0.0000025980 & -0.0000008135 & 0 & & & & & & \\
\hline & & & & & 0 & -0.0000011040 & -0.0000008135 & 0.0000010811 & 0 & & & & & & \\
\hline & & & & & -0.0000006854 & 0 & 0 & 0 & 0.0000009054 & & & & & & \\
\hline & & & & & inverse Jacobian & & & & & & {$[\mathrm{F}]$} & & {$[\Delta x]$} & & \\
\hline & & & & & 884135.2837 & 744293.124 & 668819.3565 & 503247.8269 & 669267.4931 & & -0.0000288743 & & -15.64678921 & & \\
\hline & & & & & 744293.124 & 764623.2528 & 687087.9435 & 516993.8804 & 563410.6029 & & -0.0000032833 & & -12.4224294 & & \\
\hline & & & & & 668819.3565 & 687087.9435 & 1120956.442 & 843454.795 & 506278.9172 & & 0.0000001763 & & -6.280508448 & & \\
\hline & & & & & 503247.8269 & 516993.8804 & 843454.795 & 1559632.545 & 380945.5608 & & 0.0000126515 & & 6.976695969 & & \\
\hline & & & & & 669267.4931 & 563410.6029 & 506278.9172 & 380945.5608 & 1611104.18 & & 0.0000087274 & & -2.204883833 & & \\
\hline
\end{tabular}

"from the previous iteration

Dejan Brkic, Strumicka 88, 11050 Belgrade, Serbia; dejanrgf@tesla.rcub.bg.ac.rs, dejanbrkic0611@gmail.com 
Standard procedure (pressure drop independent Jacobian); Re=Re(Q)
Iteration 9

\begin{tabular}{|c|c|c|c|c|c|c|c|c|c|c|c|c|c|c|c|}
\hline $\begin{array}{l}\text { Node } \\
\end{array}$ & Pipe & Length $(\mathrm{m})$ & Diameter $(\mathrm{m})$ & Pressure drop $(\mathrm{Pa})^{*}$ & Sign & Velocity $(\mathrm{m} / \mathrm{s})$ & $\mathrm{Re}$ & $\varepsilon / \mathrm{D}$ & Colebrook: $\lambda^{-0.5}$ & $\lambda$ & $Q\left(\mathrm{~m}^{3} / \mathrm{s}\right)$ & $|\partial \alpha / \partial(\Delta p)|$ & correction 1 (Pa) & correction 2 (Pa) & pressure drop $\mathrm{Pa}$ \\
\hline 1 & $\frac{12}{12}$ & 400 & 0.3526 & -7985 & -1 & 1.00 & 350374.55 & 0.0000085082 & 8.407808403 & 0.014146024 & -0.09741 & 0.0000060997 & 11.27034377 & -9.764773828 & \\
\hline \multirow[t]{3}{*}{1} & \multirow[t]{3}{*}{16} & \multirow[t]{3}{*}{900} & \multirow[t]{3}{*}{0.2204} & -23778 & \multirow[t]{3}{*}{-1} & \multirow[t]{3}{*}{0.85} & \multirow[t]{3}{*}{187641.76} & \multirow[t]{3}{*}{0.0000136116} & 7.920191301 & 0.015941481 & -0.03261 & \multirow{2}{*}{0.0000006857} & \multirow[t]{3}{*}{11.27034377} & \multirow[t]{3}{*}{-1.473030063} & \multirow{3}{*}{$\begin{array}{l}-2983 \\
-23768\end{array}$} \\
\hline & & & & & & & & & \multirow{2}{*}{\multicolumn{3}{|c|}{$\begin{array}{lc} & 0.13000 \\
\text { stopping criterion } \Sigma=0 ; \Sigma & -0.000015901479 \\
\end{array}$}} & & & & \\
\hline & & & & & & & & & & & & 0.0000067854 & & & \\
\hline 2 & 21 & 400 & 0.3526 & 7985 & 1 & 1.00 & 350374.55 & 0.0000085082 & 8.407808403 & 0.014146024 & 0.09741 & 0.0000060997 & 9.764773828 & -11.27034377 & 7983 \\
\hline 2 & 23 & 500 & 0.2468 & -11347 & -1 & 0.85 & 208205.99 & & 8.00213906 & 0.015616648 & -0.04052 & \multirow{3}{*}{$\begin{array}{l}0.00000017854 \\
0.0000009652\end{array}$} & \multirow{4}{*}{$\begin{array}{l}9.764773828 \\
9.764773828\end{array}$} & $\begin{array}{l}-5.665890179 \\
0\end{array}$ & -11343 \\
\hline \multirow[t]{3}{*}{2} & \multirow[t]{3}{*}{25} & \multirow[t]{3}{*}{900} & \multirow{3}{*}{$\begin{array}{l}0.2408 \\
0.2468\end{array}$} & -21704 & \multirow[t]{3}{*}{-1} & \multirow{3}{*}{0.88} & \multirow{3}{*}{$\begin{array}{l}215200.99 \\
215309.93\end{array}$} & \multirow{3}{*}{$\begin{array}{l}0.00000121556 \\
0.0000121556\end{array}$} & 8.027635962 & 0.015517604 & -0.04190 & & & & -21694 \\
\hline & & & & & & & & & stonni & g criterion $\Sigma=0 \cdot \Sigma$ & $\begin{array}{c}-0.01500 \\
-0.000007559800\end{array}$ & & & & \\
\hline & & & & & & & & & & & & 0.0000088502 & & & \\
\hline 3 & 32 & 500 & 0.2468 & 11347 & 1 & 0.85 & 20820599 & 0.0000121556 & 8.00213906 & 0.015616648 & 0.04052 & 0.0000017854 & 5,665890179 & $-9,764773828$ & 11343 \\
\hline 3 & 34 & 800 & 0.2204 & -18745 & -1 & 0.80 & 175605.01 & 0.0000136116 & 7.86984233 & 0.016146111 & -0.03051 & 0.0000008139 & 5.665890179 & 1.614375179 & -18738 \\
\hline & & & & & & & & & ctonni & Geritorin $5-0$. & $\begin{array}{c}-0.01000 \\
\end{array}$ & & & & \\
\hline & & & & & & & & & Stoppi & $g$ Cinterion $L-0, L$ & & 00000025993 & & & \\
\hline 4 & 43 & 800 & 0.2204 & 18745 & 1 & 0.80 & 175605.01 & 0.0000136116 & 7.86984233 & 0.016146111 & 0.03051 & 0.0000008139 & -1.614375179 & -5.665890179 & 18738 \\
\hline 4 & 45 & 400 & 0.1102 & 8388 & 1 & 0.47 & 51710.89 & 0.0000272232 & 6.927823091 & 0.02083562 & 0.00449 & 0.0000002678 & -1.614375179 & & 8386 \\
\hline & & & & & & & & & stonni & o criterion $S=0.5$ & $\begin{array}{c}-0.03500 \\
0.00635778\end{array}$ & & & & \\
\hline 5 & omitted to & linear indenp & cy of Jacobian & & & & & & & & & & & & \\
\hline 6 & 61 & 900 & 0.2204 & 23778 & 1 & 0.85 & 187641.76 & $1.36116 \mathrm{E}-05$ & 7.920191301 & 0.015941481 & 0.03261 & 0.0000006857 & 1.473030063 & -11.27034377 & 23768 \\
\hline 6 & 65 & 400 & 0.0968 & -5911 & -1 & 0.35 & 34092.67 & 3.09917E-05 & 6.610663188 & 0.022882841 & -0.00260 & 0.0000002201 & 1.473030063 & & -5910 \\
\hline & & & & & & & & & ctonni & G criterinon $S=0.5$ & $\frac{-0.03000}{0.0039787}$ & & & & \\
\hline & & & & & & & & & & & 0.000006393787 & 0.0000009058 & & & \\
\hline & & & & & Jacobian & & & & & & & & & & \\
\hline & & & & & 0.0000067854 & -0.0000060997 & 0 & 0 & -0.0000006857 & & & & & & \\
\hline & & & & & -0.0000060997 & 0.0000088502 & -0.0000017854 & 0 & 0 & & & & & & \\
\hline & & & & & 0 & -0.0000017854 & 0.0000025993 & -0.0000008139 & 0 & & & & & & \\
\hline & & & & & & 0 & -0.0000008139 & 0.0000010817 & 0 & & & & & & \\
\hline & & & & & -0.0000006857 & 0 & & 0 & 0.0000009058 & & & & & & \\
\hline & & & & & inverse Jacobian & & & & & & [F] & & {$[\Delta x]$} & & \\
\hline & & & & & 883726.3969 & 743923.4822 & 668482.0413 & 502997.3527 & 668982.3671 & & -0.0000159015 & & -11.27034377 & & \\
\hline & & & & & 743923.4822 & 764245.1827 & 686742.9138 & 516737.6927 & 563151.3258 & & -0.0000075598 & & -9.764773829 & & \\
\hline & & & & & 668482.0413 & 686742.9138 & 1120411.02 & 843049.9879 & 506042.0283 & & 0.0000013925 & & -5.665890179 & & \\
\hline & & & & & 502997.3527 & 516737.6927 & 843049.9879 & 1558832.824 & 380769.841 & & 0.0000063578 & & 1.614375178 & & \\
\hline & & & & & 668982.3671 & 563151.3258 & 506042.0283 & 380769.841 & 1610404.839 & & 0.0000063938 & & -1.473030064 & & \\
\hline
\end{tabular}




\begin{tabular}{|c|c|c|c|c|c|c|c|c|c|c|c|c|c|c|c|}
\hline Node & Pipe & Length $(\mathrm{m})$ & Diameter $(m)$ & Pressure drop (Pa) & Sign & Velocity $(\mathrm{m} / \mathrm{s})$ & $\operatorname{Re}$ & $\varepsilon / D$ & Colebrook: ${ }^{-0.5}$ & $\lambda$ & $Q\left(\mathrm{~m}^{3} / \mathrm{s}\right)$ & $|\partial \alpha / \partial(\Delta p)|$ & correction $1(\mathrm{~Pa})$ & correction $2(\mathrm{~Pa})$ & pressure drop $(\mathrm{Pa}$ \\
\hline & 12 & 400 & 0.3526 & -7983 & -1 & & 350336.58 & 0.0000085082 & 8.407725945 & 0.014146301 & -0.09740 & 0.0000061002 & -0.401422549 & 0.24529831 & -7983 \\
\hline \multirow[t]{3}{*}{1} & \multirow[t]{3}{*}{16} & \multirow[t]{3}{*}{900} & \multirow[t]{3}{*}{0.2204} & -23768 & \multirow[t]{3}{*}{-1} & \multirow[t]{3}{*}{0.85} & \multirow[t]{3}{*}{187627.86} & \multirow[t]{3}{*}{0.0000136116} & 7.920135052 & 0.015941707 & -0.03260 & 0.0000006858 & $\begin{array}{l}-0.401422549 \\
-0.40259\end{array}$ & 0.058059084 & -23768 \\
\hline & & & & & & & & & \multirow{2}{*}{\multicolumn{3}{|c|}{$\begin{array}{ll}\text { stopping criterion } \Sigma=0 ; \Sigma & 0.000001187876 \\
\end{array}$}} & \multirow{2}{*}{\multicolumn{2}{|c|}{0.0000067860}} & & \\
\hline & & & & & & & & & & & & & & & \\
\hline 2 & 21 & 400 & 0.3526 & 7983 & 1 & 1.00 & 350336.58 & 0.0000085082 & 8.407725945 & 0.014146301 & 0.09740 & 0.0000061002 & -0.24529831 & 0.401422549 & 7983 \\
\hline 2 & 23 & 500 & 0.2468 & -11343 & -1 & 0.85 & & & 8.002070061 & 0.015616917 & -0.04051 & \multirow{3}{*}{$\begin{array}{l}0.0000017857 \\
0.0000009654\end{array}$} & \multirow{4}{*}{$\begin{array}{r}-0.24529831 \\
-0.24529831\end{array}$} & 0.045310755 & -11343 \\
\hline \multirow[t]{3}{*}{2} & \multirow[t]{3}{*}{25} & \multirow[t]{3}{*}{900} & \multirow{3}{*}{0.2468} & -21694 & \multirow{3}{*}{$\begin{array}{l}-1 \\
-1\end{array}$} & \multirow{3}{*}{0.88} & \multirow{3}{*}{215296.56} & \multirow{3}{*}{0.0000121556} & 8.027588783 & 0.015517786 & -0.04189 & & & & \multirow{3}{*}{$\begin{array}{l}-11345 \\
-21695\end{array}$} \\
\hline & & & & & & & & & \multirow{2}{*}{\multicolumn{3}{|c|}{\begin{tabular}{ll|} 
stopping criterion $\Sigma=0 ; \Sigma$ & -0.000000358452 \\
\end{tabular}}} & \multirow{2}{*}{\multicolumn{2}{|c|}{0.0000088513}} & & \\
\hline & & & & & & & & & & & & & & & \\
\hline 3 & 32 & 500 & 0.2468 & 11343 & 1 & 0.85 & 208187.09 & 0.0000121556 & 8.002070061 & 0.015616917 & 0.04051 & 0.0000017857 & -0.045310755 & 0.24529831 & 11343 \\
\hline \multirow[t]{3}{*}{3} & 34 & 800 & 0.2204 & -18738 & -1 & 0.80 & 175576.86 & 0.0000136116 & 7.869720474 & 0.016146611 & -0.03051 & 0.0000008141 & -0.045310755 & -0.544487718 & -18739 \\
\hline & & & & & & & & & stopp & $\bar{g}$ criterion $\sum=0 ; \varepsilon$ & -0.01000 & & & & \\
\hline & & & & & & & & & & & & 0.0000025997 & & & \\
\hline 4 & 43 & 800 & 0.2204 & 18738 & 1 & 0.80 & 175576.86 & 0.0000136116 & 7.869720474 & 0.016146611 & 0.03051 & 0.0000008141 & 0.544487718 & 0.045310755 & 18739 \\
\hline 4 & 45 & 400 & 0.1102 & 8386 & 1 & 0.47 & 51694.78 & 0.0000272232 & 6.927587182 & 0.020837039 & 0.00449 & 0.0000002678 & 0.544487718 & & 8387 \\
\hline & & & & & & & & & stopd & g criterion $\Sigma=0: \Sigma$ & $\begin{array}{c}-0.0003500 \\
-0.0000025939\end{array}$ & & & & \\
\hline 5 & omitted to $\mathrm{p}$ & near indenp & cy of Jacobian & & & & & & & & & & & & \\
\hline 6 & 61 & 900 & 0.2204 & 23768 & 1 & 0.85 & 187627.86 & 0.0000136116 & 7.920135052 & 0.015941707 & 0.03260 & 0.0000006858 & -0.058059084 & 0.401422549 & 23768 \\
\hline 6 & 65 & 400 & 0.0968 & -5910 & -1 & 0.35 & 34091.59 & 0.0000309917 & 6.610639052 & 0.022883008 & -0.00260 & 0.0000002201 & -0.058059084 & & -5910 \\
\hline & & & & & & & & & & & -0.03000 & & & & \\
\hline & & & & & & & & & stopp & g criterion $\Sigma=0 ; \Sigma$ & -0.000000222710 & & & & \\
\hline & & & & & Jacobian & & & & & & & 0.0000009060 & & & \\
\hline & & & & & 0.0000067860 & -0.0000061002 & 0 & 0 & -0.0000000858 & & & & & & \\
\hline & & & & & -0.0000061002 & 0.0000088513 & -0.0000017857 & 0 & & & & & & & \\
\hline & & & & & & -0.0000017857 & 0.0000025997 & -0.0000008141 & 0 & & & & & & \\
\hline & & & & & & & -0.0000008141 & 0.0000010818 & & & & & & & \\
\hline & & & & & -0.0000006858 & 0 & 0 & $\begin{array}{l}0 \\
0\end{array}$ & 0.0000009060 & & & & & & \\
\hline & & & & & inverse Jacobian & & & & & & [F] & & {$[\Delta x]$} & & \\
\hline & & & & & 883571.1344 & 743779.1659 & 668357.8493 & 502918.4147 & 668877.5103 & & 0.0000011879 & & 0.40142255 & & \\
\hline & & & & & 743779.1659 & 764098.0505 & 686616.3414 & 516657.3599 & 563052.7496 & & -0.0000003585 & & 0.245298311 & & \\
\hline & & & & & 668357.8493 & 686616.3414 & 1120219.906 & 842930.5044 & 505957.6041 & & 0.0000001230 & & 0.045310757 & & \\
\hline & & & & & 502918.4147 & 516657.3599 & 842930.5044 & 1558622.659 & 380717.3005 & & -0.0000006259 & & -0.544487717 & & \\
\hline & & & & & 668877.5103 & 563052.7496 & 505957.6041 & 380717.3005 & 1610136.352 & & -0.00000002227 & & 0.058059085 & & \\
\hline
\end{tabular}

*from the previous iteration

Def 
Standard procedure (pressure drop independent Jacobian); Re=Re(0)

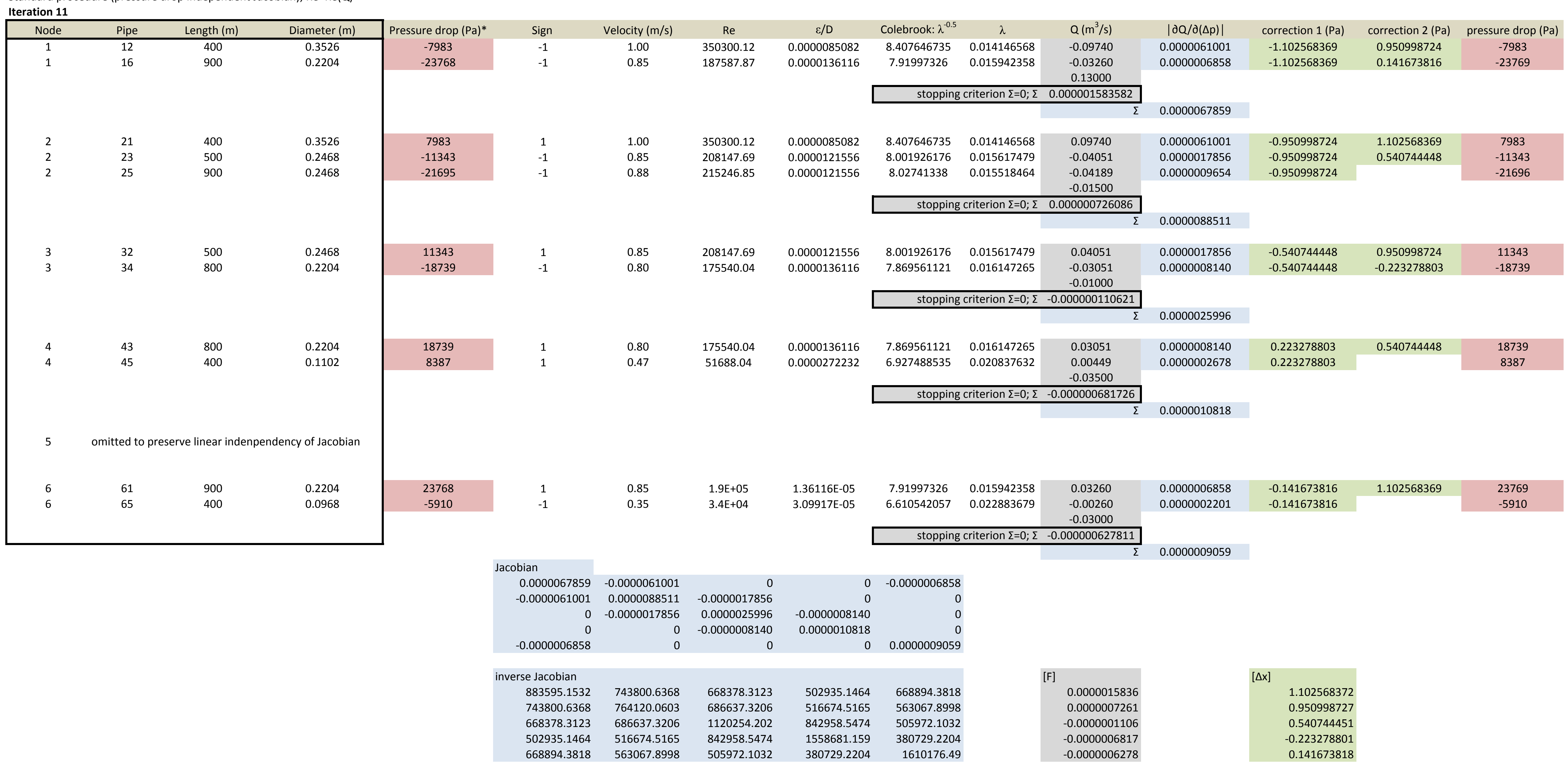

*from the previous iteration

(n)

Belgrade, Serbia; dejanrgf@tesla.rcub.bg.ac.rs, dejanbrkic0611@gmail.com 


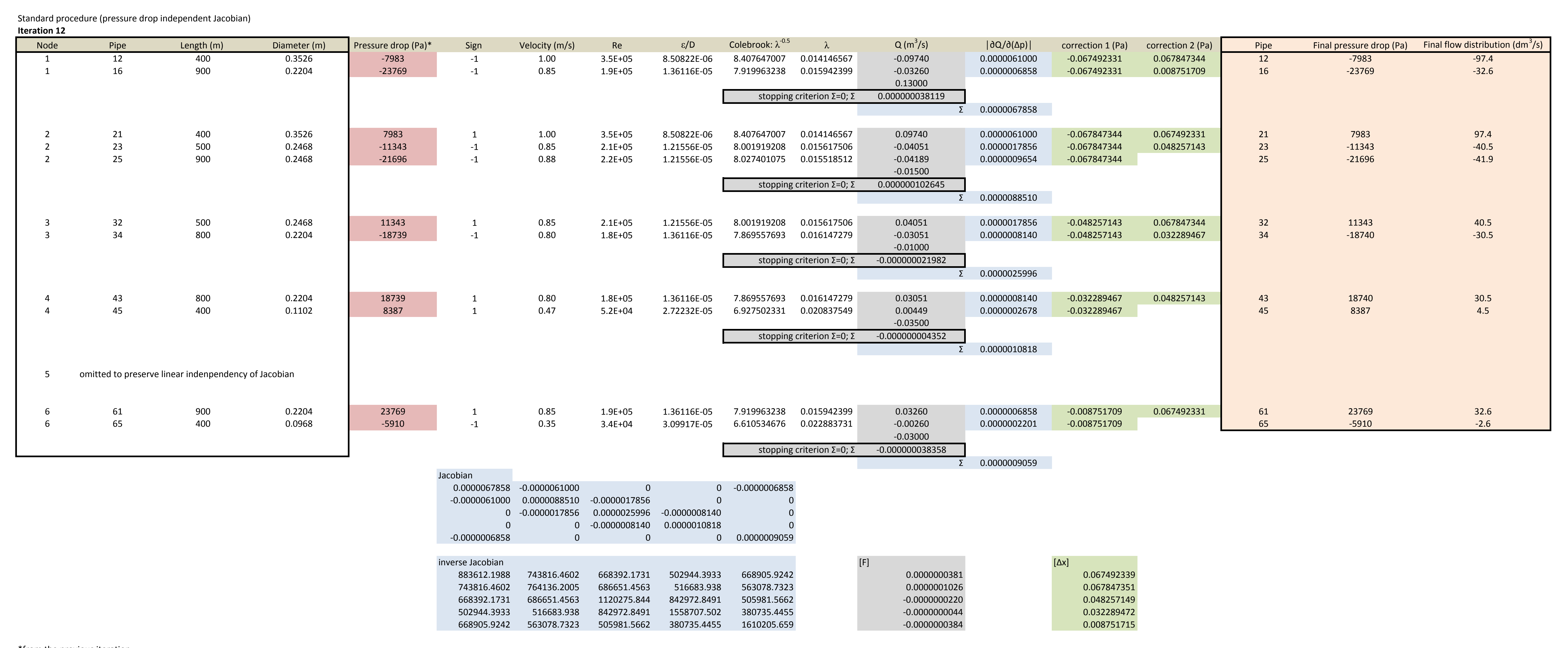


Figure caption list

Figure 1. Convergence properties of the proposed method for the solution of the problem of

water distribution analysis expressed in the nodal form (both with the independent Jacobian)

Etable 1.

Etable 2.

(Etable 1 and Etable 2 are submitted as Electronic Annexes to the discussion; do not embed them into the text, instead provide a link to this material) 


\section{ASTEII: \\ COPYRIGHT TRANSFER AGREEMENT}

Manuscript Number

Type: Discussion

DiSCUSSION OF "WATERDISTRIBUTION SYSTEM ANALYSIS: NEWTONPublication'Title: RAPHSON METMOD REVISITED" BY M. SPILIOTIS AND G. TSAKIRIS Manuscript Authors: DEJAN BRKIĆ Corresponding Author Name and Address: DEJANBRKIC' STRUMIČKA 88,11050 BELGRADE, SERBIA

This form must ${ }^{*}$ be returned with your final manuscript to: American Society of Civil Engineers. Journals Production Services Dept., 1801 Alexander Bell Drive, Reston, VA 20191-4400.

The author(s) warrant(s) that the above cited manuscript is the original work of the author(s) and has never been published in its present form.

The undersigned, with the consent of all authors, hereby transfers, to the extent that there is copyright to be transferred, the exclusive copyright interest in the above-cited manuscript (subsequently called the "work"), in this and all subsequent editions of this work, and in derivatives, translations, or ancillaries, in English and in foreign translations, in all formats and media of expression now known or later developed, including electronic, to the American Society of Civil lingineers subject to the following.

- The undersigned author and all coauthors retain the right to revise, adapt, prepare derivative works, present orally, or distribute the work provided that all such use is for the personal nonconmercial benefit of the authori(s) and is consistent with any prior contractual agreement between the undersigned and/or coauthors and their employer(s).

- In all instances where the work is prepared as a "work made for hire" for an employer, the employer(s) of the author(s) retain(s) the right to revise, adapt, prepare derivative works, publish, reprint, reproduce, and distribute the work provided that such use is for the promotion of its business enterprise and coes not imply the endorsement of ASCE.

- No proprietary right other than copyright is claimed by ASCE.

- An author who is a U.S. Government employee and prepared the above-cited work does not own copyright in it. If at least one of the authors is not in this category, that author should sign below. If all the authors are in this category, check here $\square$ and sign here:

Please return this form by mail.

SIGN HERE FOR COPYRIGHT TRANSFER [Individual Author or Employer's Authorized Agent (work made for hire)]

Print Author's Name: DEJAN BRKIC

Print Agent's Name and Title:
Signature of Author (in ink):

Signature of Agency Rep (in ink):

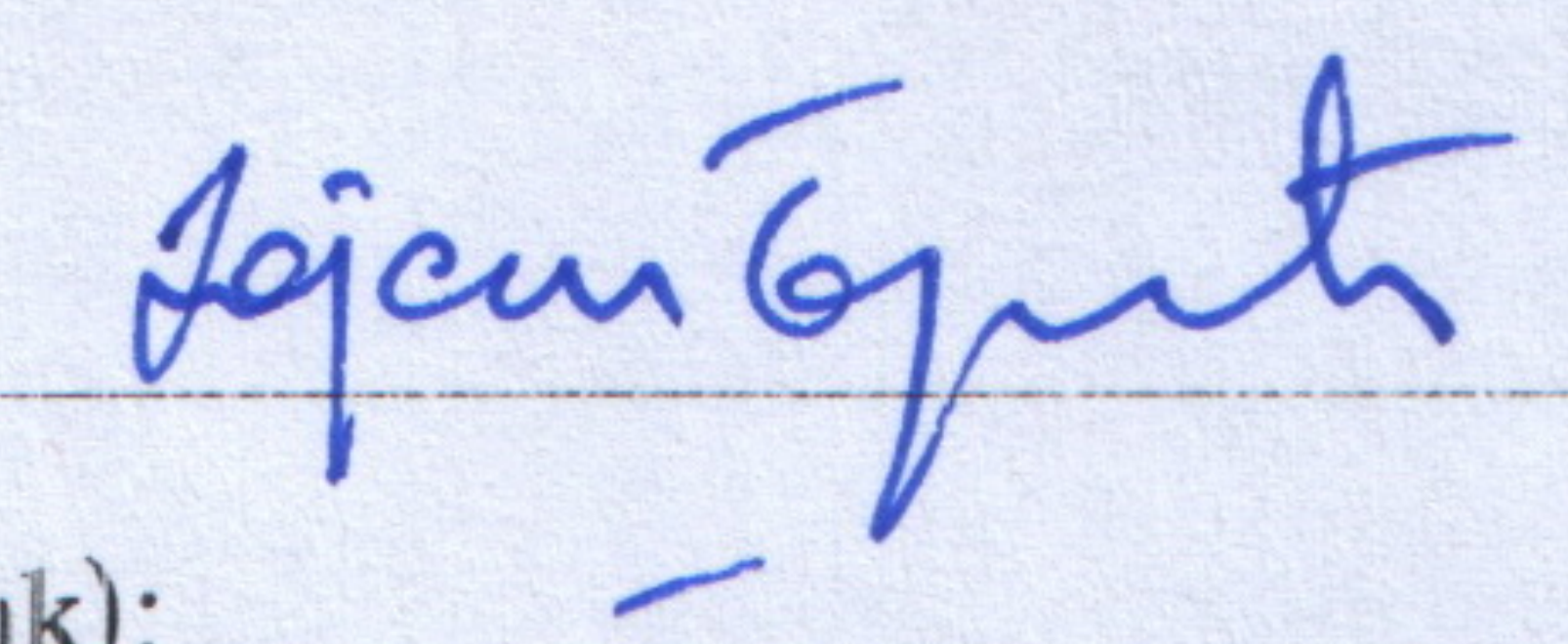

Date: 06. DECEMBER 2011

Note: If the manuscript is not accepted by ASCE or is withdrawn prior to acceptance by ASCE, this transfer will be null and void and the form will be returned to the author. 
Dear Editor/Reviewers,

I have accepted all your suggestions. I am not native English speaker and therefore I appreciate your grammar corrections of my text. Thank you!

Additional request for tables: I do not have any tables in my text; (Etable 1 and Etable 2 are submitted as Electronic Annexes to the discussion; do not embed them into the text, instead provide a link to this material). This is possible for technical papers, technical notes and case studies since there is separate item description in editorial system for such purposes. This item description does not exist for discussions. So I have uploaded them as figures. Please, advise me if you have additional requests with these materials.

Sincerely yours,

Dejan Brkić, Ph.D. 